\title{
Scenario-based cuts for structured two-stage stochastic and distributionally robust $p$-order conic mixed integer programs
}

\author{
Manish Bansal ${ }^{1}$ (D) Y Yingqiu Zhang ${ }^{1}$
}

Received: 23 February 2020 / Accepted: 26 December 2020

(c) The Author(s), under exclusive licence to Springer Science+Business Media, LLC part of Springer Nature 2021

\begin{abstract}
In this paper, we derive (partial) convex hull for deterministic multi-constraint polyhedral conic mixed integer sets with multiple integer variables using conic mixed integer rounding (CMIR) cut-generation procedure of Atamtürk and Narayanan (Math Prog 122:1-20, 2008), thereby extending their result for a simple polyhedral conic mixed integer set with single constraint and one integer variable. We then introduce two-stage stochastic $p$-order conic mixed integer programs (denoted by TSS-CMIPs) in which the second stage problems have sum of $l_{p}$-norms in the objective function along with integer variables. First, we present sufficient conditions under which the addition of scenario-based nonlinear cuts in the extensive formulation of TSS-CMIPs is sufficient to relax the integrality restrictions on the second stage integer variables without impacting the integrality of the optimal solution of the TSSCMIP. We utilize scenario-based CMIR cuts for TSS-CMIPs and their distributionally robust generalizations with structured CMIPs in the second stage, and prove that these cuts provide conic/linear programming equivalent or approximation for the second stage CMIPs. We also perform extensive computational experiments by solving stochastic and distributionally robust capacitated facility location problem and randomly generated structured TSS-CMIPs with polyhedral CMIPs and second-order CMIPs in the second stage, i.e. $p=1$ and $p=2$, respectively. We observe that there is a significant reduction in the total time taken to solve these problems after adding the scenario-based cuts.
\end{abstract}

Keywords Two-stage stochastic $p$-order conic mixed integer program $\cdot$ Scenario-based cutting planes · Two-stage distributionally robust program · (Partial) convex hull $\cdot$ Conic mixed integer rounding $\cdot$ Multi-module capacitated facility location

$凶$ Manish Bansal

bansal@vt.edu

Yingqiu Zhang

yqzhang7@vt.edu

1 Department of Industrial and Systems Engineering, Virginia Tech, 1145 Perry Street, Blacksburg, VA 24060, USA 


\section{Introduction}

In this paper, we consider two-stage stochastic $p$-order conic mixed integer programs (TSSCMIPs) in which the first stage has pure integer variables and the second stage problems have sum of $l_{p}$-norms in the objective function along with integer variables. More specifically, the TSS-CMIP is defined as follows:

$$
\min \left\{c^{\top} x+\mathbb{E}_{\xi_{P}}\left[\overline{\mathcal{Q}}_{\omega}(x)\right]: A x \geq b, x \in \mathbb{Z}^{n_{1}}\right\},
$$

where random variable $\xi_{P}$ follows a known probability distribution $P$ with a finite sample space $\Omega$, and for scenario $\omega \in \Omega$ with $\bar{p}_{\omega}$ probability of occurrence:

$$
\begin{gathered}
\overline{\mathcal{Q}}_{\omega}(x):=\min \left\{g_{\omega}^{\top} y_{\omega}+\sum_{j \in J} \hat{g}_{\omega}^{j}\left\|E_{\omega}^{j} y_{\omega}^{j}+F_{\omega}^{j} x-h_{\omega}^{j}\right\|_{p}:\right. \\
\left.W_{\omega} y_{\omega} \geq r_{\omega}-T_{\omega} x, y_{\omega}^{j} \in \mathbb{Z}^{q}, j \in J\right\},
\end{gathered}
$$

which is equivalent to

$$
\begin{gathered}
\overline{\mathcal{Q}}_{\omega}(x):=\min g_{\omega}^{\top} y_{\omega}+\sum_{j \in J} \hat{g}_{\omega}^{j} d_{\omega, 0}^{j} \\
\text { s.t. } W_{\omega} y_{\omega} \geq r_{\omega}-T_{\omega} x, \quad\left\|E_{\omega}^{j} y_{\omega}^{j}+F_{\omega}^{j} x-h_{\omega}^{j}\right\|_{p} \leq d_{\omega, 0}^{j}, j \in J, \\
\quad y_{\omega}^{j} \in \mathbb{Z}^{q}, d_{\omega, 0}^{j} \in \mathbb{R}_{+}, j \in J .
\end{gathered}
$$

Here, $\|\cdot\|_{p}$ denotes $l_{p}$-norm, i.e. $\|\mathbf{y}\|_{p}=\left(\sum_{k}\left|y_{k}\right|^{p}\right)^{1 / p}$ for $p \geq 1, c \in \mathbb{R}^{n_{1}}, A \in \mathbb{R}^{m_{1} \times n_{1}}$, $b \in \mathbb{R}^{m_{1}}$, and for each $\omega \in \Omega$ and $j \in J:=\{1, \ldots,|J|\}, g_{\omega} \in \mathbb{R}^{q|J|}, \hat{g}_{\omega}^{j} \in \mathbb{R}, E_{\omega}^{j} \in \mathbb{R}^{m_{2} \times q}$, $F_{\omega}^{j} \in \mathbb{R}^{m_{2} \times n_{1}}, h_{\omega}^{j} \in \mathbb{R}^{m_{2}}, W_{\omega} \in \mathbb{R}^{m_{3} \times q|J|}, T_{\omega} \in \mathbb{R}^{m_{3} \times n_{1}}$, and $r_{\omega} \in \mathbb{R}^{m_{3}}$. We refer to the formulation (2)-(4) and function $\overline{\mathcal{Q}}_{\omega}(x)$ as the second stage subproblem and the recourse function, respectively. For TSS-CMIPs, we make the following assumptions:

(A1) $T_{\omega} \in \mathbb{Z}^{m_{3} \times n_{1}}$ and $F_{\omega}^{j} \in \mathbb{Z}^{m_{2} \times n_{1}}$ for all $\omega \in \Omega$ and $j \in J$ (w.l.o.g.),

(A2) $X:=\left\{x: A x \geq b, x \in \mathbb{Z}^{n_{1}}\right\}$ is non-empty,

(A3) Relatively complete recourse, i.e. $\overline{\mathcal{K}}_{\omega}(x):=\left\{\left(y_{\omega}, d_{\omega, 0}\right):(3)-(4)\right\} \neq \emptyset$ for $(x, \omega) \in$ $X \times \Omega$.

The deterministic equivalent (also referred to as extensive formulation) of TSS-CMIP (1) is a large-scale deterministic CMIP, defined by

$$
\begin{aligned}
& \min \left\{c x+\sum_{\omega \in \Omega} \bar{p}_{\omega}\left(g_{\omega} y_{\omega}+\sum_{j \in J} \hat{g}_{\omega}^{j} d_{\omega, 0}^{j}\right):\right. \\
& (x, y, d) \in \mathbb{Z}^{n_{1}} \times\left(\mathbb{Z}^{q_{1}} \times \mathbb{R}^{q-q_{1}}\right)^{|\Omega| \times|J|} \times \mathbb{R}_{+}^{|\Omega| \times|J|}, \\
& A x \geq b, \quad T_{\omega} x+W_{\omega} y_{\omega} \geq r_{\omega}, \omega \in \Omega, \\
& \left.\left\|F_{\omega}^{j} x+E_{\omega}^{j} y_{\omega}^{j}-h_{\omega}^{j}\right\|_{p} \leq d_{\omega, 0}^{j}, j \in J, \omega \in \Omega\right\} .
\end{aligned}
$$

We present sufficient conditions under which by adding scenario-based nonlinear cuts in $\left(x, y_{\omega}, d_{\omega, 0}\right)$ space to the extensive formulation, the integrality constraints on the $y_{\omega}$ integer variables can be relaxed without impacting the integrality of the optimal solution of the 
problem. In other words, we derive partial convex hull (see Definition 1) for the deterministic equivalent of TSS-CMIP. For the following structured TSS-CMIPs, we derive scenario-based cuts that satisfy these conditions:

(a) $E_{\omega}=\mathbf{I}_{2}$ (identity matrix of size $2 \times 2$ ), $F_{\omega}^{j}=[1,0]^{T}$ for $j \in J$, and $m_{3}=0$;

(b) $E_{\omega}^{j}=\mathbf{1}$ (vector of all ones) for $j \in J$ and $W_{\omega}$ is totally unimodular (TU);

(c) $E_{\omega}^{j}$ for all $j \in J$, and $W_{\omega}$ are network flow matrices;

(d) $E_{\omega}^{j}$ for all $j \in J$ are TU matrices, and $W_{\omega}=\mathbf{I}_{m_{3}}$ or $W_{\omega}=\mathbf{0}$.

We prove that these cuts provide conic programming equivalent for the structured CMIPs in the second stage of (a) with $p=2$ and (b-d) with $p=1$, and conic programming approximation for (b-d) with $p=2$. Note that in (b-d), there is no restriction on matrices $F_{\omega}^{j}$ for $j \in J$ and $\omega \in \Omega$. Moreover, we are considering TU matrices in the aforementioned structured CMIPs because of the following reasons: (1) In the literature on TSS-MILPs, researchers have considered two-stage stochastic mixed integer linear programs (TSS-MILPs) with TU recourse matrix [51] and extensive formulation of TSS-MILPs and multi-stage stochastic MIPs with TU constraint matrix [32,59]; (2) TU matrices also find their applications in deterministic problems such as two-commodity transportation problem [49], network flow model for nursing staffs' scheduling problems [27], and many more [42]; and (3) TSS-CMIPs and their distributionally robust variants (see Sect. 1.2) with these structured CMIPs have not been studied in the literature.

In the following subsections, we discuss other contributions of this paper, in particular (partial) convex hulls for deterministic multi-constraint polyhedral conic mixed integer sets with multiple integer variables, conic/linear programming equivalent or approximation for structured CMIPs in the second stage of TSS-CMIPs and their distributionally robust generalizations, and application of the foregoing results for solving stochastic capacitated facility location problem.

Definition 1 A partial convex hull of a conic mixed integer set $\mathfrak{N}$ is another conic mixed integer set $\mathfrak{N}_{\text {pch }}$ such that $\mathfrak{N} \subseteq \mathfrak{N}_{\text {pch }} \subseteq \operatorname{conv}(\mathfrak{N})=\operatorname{conv}\left(\mathfrak{N}_{\text {pch }}\right)$. Note that $\mathfrak{N}_{\text {pch }}$ has lesser number of integrality constraints (but possibly more linear or nonlinear inequalities) than $\mathfrak{N}$. Whereas in comparison to conv $(\mathfrak{N}), \mathfrak{N}_{\text {pch }}$ might have lesser inequalities but more integrality constraints.

\subsection{Deterministic polyhedral conic mixed integer sets}

Atamtürk and Narayanan [3] generalize the well-known mixed integer rounding (MIR) inequalities of Nemhauser and Wolsey [43] by studying a polyhedral (or first-order) conic mixed integer set defined by a single conic constraint and one integer variable, i.e., $Z^{1,1}:=\left\{\left(\sigma, \rho_{1}\right) \in \mathbb{Z} \times \mathbb{R}_{+}:|\sigma-\beta| \leq \rho_{1}\right\}$. They introduce so-called conic MIR cut for $Z^{1,1}$ which is facet-defining for the convex hull of $Z^{1,1}$, denoted by $\operatorname{con} v\left(Z^{1,1}\right)$. The conic MIR cut along with defining and nonnegativity constraints are sufficient to describe $\operatorname{conv}\left(Z^{1,1}\right)$. Moreover, they demonstrate how this cut can be utilized to develop strong cutting planes for general conic mixed integer programs (CMIPs), thereby providing an effective approach to solve them. We introduce four new deterministic multi-constraint polyhedral conic mixed integer sets with multiple integer variables, derive conic MIR cuts for them, and provide conditions under which the addition of these cuts is sufficient to provide the convex hull or a partial convex hull of the sets. More specifically, we study the following generalizations of the set $Z^{1,1}$ : 
(i) Set I:

$$
\begin{aligned}
S_{K}^{m, n}: & =\left\{\left(\sigma, \rho, \rho_{0}\right) \in \mathbb{Z}^{n K} \times \mathbb{R}_{+}^{m K} \times \mathbb{R}_{+}^{K}: \mathcal{A} \sigma \geq b,\right. \\
& \left.\left|\sum_{t=1}^{n} g_{k t}^{i} \sigma_{k t}-\beta_{i k}\right| \leq \rho_{i}^{k}, i=1, \ldots, m, k=1, \ldots, K\right\}
\end{aligned}
$$

where $\beta \in \mathbb{R}^{m K}, \mathcal{A} \in \mathbb{R}^{m_{3} \times n K}$ and $\mathcal{G}^{k}=\left(g_{k t}^{i}\right) \in \mathbb{R}^{m \times n}$ for $k=1, \ldots, K$.

(ii) Set II:

$$
\begin{aligned}
U_{K}^{m, n, u}: & =\left\{\left(\eta, \sigma, \rho, \rho_{0}\right) \in \mathbb{Z}^{u} \times \mathbb{Z}^{n K} \times \mathbb{R}_{+}^{m K} \times \mathbb{R}_{+}^{K}: \mathcal{A}_{1} \eta+\mathcal{A} \sigma \geq b,\right. \\
& \left.\left\|\rho^{k}\right\|_{1} \leq \rho_{0}^{k}, \quad\left|\sum_{t=1}^{u} c_{k t}^{i} \eta_{t}+\sum_{t=1}^{n} g_{k t}^{i} \sigma_{k t}-\beta_{i k}\right| \leq \rho_{i}^{k}, i=1, \ldots, m, k=1, \ldots, K\right\}
\end{aligned}
$$

where $\beta \in \mathbb{R}^{m K}, \mathcal{A} \in \mathbb{R}^{m_{3} \times n K}$, and $\mathcal{G}^{k}=\left(g_{k t}^{i}\right) \in \mathbb{R}^{m \times n}$ for $k=1, \ldots, K$.

We assume that matrices $\mathcal{A}_{1}$ and $\mathcal{C}^{k}=\left(c_{k t}^{i}\right)$, for $k=1, \ldots, K$, are integral. We also study sets $S_{K}^{m, n}$ and $U_{K}^{m, n, u}$ with $n=1$ and $g_{k 1}^{i}=1$ for all $(i, k)$.

\subsection{Structured two-stage stochastic and distributionally robust p-order conic mixed integer programs}

We introduce TSS-CMIPs with structured multi-constraint $p$-order conic mixed integer sets having multiple integer variables in the second stage. We derive scenario-based cuts for these second stage structured CMIPs using conic MIR and prove that these cuts provide a convex programming equivalent or approximation, in particular, a conic/linear program, for the second stage CMIPs. The foregoing second stage convexification results also hold for distributionally robust generalizations of TSS-CMIPs where we seek a solution that optimizes the expected value of the objective function for the worst case probability distribution within a prescribed (ambiguity) set of distributions that may be followed by the uncertain parameters $[11,14,18,22,23,26,37,46-48,53,62,64]$. The two-stage distributionally robust $p$-order conic mixed integer program (TSDR-CMIP) is defined as follows:

$$
\min \left\{c x+\max _{P \in \mathfrak{P}} \mathbb{E}_{\xi_{P}}\left[\overline{\mathcal{Q}}_{\omega}(x)\right] \mid x \in X\right\},
$$

where complete information about the probability distribution $P$ followed by the random variable $\xi_{P}$ is not known but it belongs to a set of distributions $\mathfrak{P}$ (referred to as the ambiguity set). In addition, we derive scenario-based valid inequalities for the extensive formulation of distributionally robust variants of the aforementioned structured TSS-CMIPs, i.e.,

$$
\begin{aligned}
& \min \left\{c x+\theta \mid \sum_{\omega \in \Omega} \bar{p}_{\omega}\left(g_{\omega} y_{\omega}+\sum_{j \in J} \hat{g}_{\omega}^{j} d_{\omega, 0}^{j}\right) \leq \theta, \quad\left\{\bar{p}_{\omega}\right\}_{\omega \in \Omega} \in \mathfrak{P} ;\right. \\
& \text { (6) - (8) hold }\} .
\end{aligned}
$$

For $|\mathfrak{P}|=1$, these cuts provide partial convex hull for the extensive formulation with no integrality restrictions on the second-stage integer variables. We also perform computational 
experiments to evaluate the effectiveness of scenario based cuts by solving structured TSSCMIPs where $p=1$ or $p=2$, and TSDR-CMIPs with $p=2$. We observe that after adding the scenario-based cuts, there is a significant reduction in the number of the second stage integer variables for instances where $p=1$ and tightening of second stage feasible region for instances where $p=2$, thereby leading to reduction in the total solution time taken to solve the TSS-CMIP and TSDR-CMIP instances. For instance, CPLEX 12.70 (with default settings) could not solve 85 out of 290 TSS-CMIP instances within a time limit of 3 hours and the allocated memory of 24 GB RAM. In contrast, after adding the scenario-based cuts at the root node, CPLEX could solve 82 out of these 85 (unsolvable) instances within 8.2 minutes (on average). For TSDR-CMIP instances with finite |P|, CPLEX 12.70 (with its default settings) could not solve 4 out of 9 instances within the time limit. After adding the cuts, CPLEX solved all these instances in 4.8 minutes (on average).

\subsection{Multi-module capacitated stochastic and distributionally robust facility location problem with subcontracting}

To illustrate the significance of the aforementioned structured TSS-CMIPs, we introduce a multi-module capacitated stochastic facility location problem with subcontracting (denoted as MM-SFLP-S), which is defined as follows. Given a set $P=\{1, \ldots, m\}$ of facilities, a set $P^{\prime}=\left\{1, \ldots, m^{\prime}\right\}$ of retailers, per unit transportation cost $t_{i j}$ for each $i \in P$ and $j \in P^{\prime}$, machines of capacity $\alpha_{k}, k \in\{1, \ldots, n\}$, that can be installed at facility $i \in P$ at the cost $c_{k}^{i}$, and per unit subcontracting $\operatorname{cost} g_{i}$ at facility $i \in P$, we formulate the MM-SFLP-S as a TSS-CMIP:

$$
\begin{array}{ll}
\min & \sum_{i \in P} \sum_{k=1}^{n} c_{i}^{k} x_{i k}+\sum_{i \in P} \sum_{j \in P^{\prime}} t_{i j} z_{i j}+\sum_{i \in P} g_{i} u_{i}+\sum_{\omega \in \Omega} \bar{p}_{\omega} Q_{\omega}(x, z, u) \\
\text { s.t. } & \sum_{k=1}^{n} x_{i k} \leq s_{i}, \quad i \in P, \\
& \sum_{j \in P^{\prime}} z_{i j} \leq \sum_{k=1}^{n} \alpha_{k} x_{i k}+u_{i}, \quad i \in P, \\
& \sum_{i \in P} u_{i} \leq r, \\
& x_{i k}, u_{i}, z_{i j} \in \mathbb{Z}_{+}, \quad i \in P, j \in P^{\prime}, k=1, \ldots, n,
\end{array}
$$

where $x_{i k}$ denotes the number of machines of capacity $\alpha_{k}$ installed at facility $i, z_{i j}$ denotes the number of items transported from facility $i \in P$ to retailer $j \in P^{\prime}$, and $u_{i}$ denotes the number of items subcontracted by facility $i \in P$. Here, an item represents either a single entity or a packet of uncountable commodity in liquid or powder form. Constraints (12) restrict the number of machines installed at facility $i$ to be at most $s_{i}$. Constraints (13) restrict the total number of items transported from facility $i$ to all retailers to be no more than the sum of the total capacity of installed machines and number of items subcontracted at the facility. Constraint (14) limits the total number of items subcontracted by all facilities to be at most $r$. Let $\mathcal{X}:=\{(x, z, u):(12)-(15)$ hold $\}$. The objective (11) of MM-SFLP-S is to minimize the total machines' installation cost, transportation cost, subcontracting cost, and the expected (second-stage) transportation cost, inventory cost at facilities, and penalty cost after the realization of uncertain demand. Note that the penalty cost is incurred for 
shipping both more or less than a retailer's demand because in case of supplying more than the demand, the retailer has to incur inventory cost. Specifically, for $(x, u, z) \in \mathcal{X}$ and $\omega \in \Omega$, the second-stage problem is defined by,

$$
\begin{aligned}
& Q_{\omega}(x, z, u):= \min \sum_{i \in P} w_{i n v}^{i}\left(\sum_{k=1}^{n} \alpha_{k} x_{i k}+u_{i}-\sum_{j \in P^{\prime}}\left(z_{i j}+y_{\omega}^{i j}\right)\right) \\
&+w_{s c} \sum_{j \in P^{\prime}} \sum_{i \in P} t_{i j} y_{\omega}^{i j}+w_{\text {pen }} \sum_{j \in P^{\prime}}\left|\sum_{i \in P}\left(y_{\omega}^{i j}+z_{i j}\right)-\zeta_{\omega}^{j}\right| \\
& \text { s.t. } \sum_{j \in P^{\prime}}\left(z_{i j}+y_{\omega}^{i j}\right) \leq \sum_{k=1}^{n} \alpha_{k} x_{i k}+u_{i}, i \in P, \\
& y_{\omega} \in \mathbb{Z}_{+}^{|P| \times\left|P^{\prime}\right|},
\end{aligned}
$$

where $y_{\omega}^{i j}$ denotes the number of items transported from facility $i \in P$ to retailer $j \in P^{\prime}$ after realization of demand $\zeta_{\omega}^{j} \geq 0$ in response to a last minute order and constraints (17) ensure that the total number of items (including the last minute order) transported from each facility to all retailers is not greater than the sum of total capacity of machines installed at the facility and number of items subcontracted from the facility. We denote per unit inventory cost at facility $i$ by $w_{i n v}^{i}$, per unit surcharge on transportation cost for any last minute order by $w_{s c}$, and per unit penalty cost for either falling short of a retailer's demand or supplying more than the demand (thereby incurring inventory cost for the retailer) by $w_{\text {pen }}$. The second-stage objective (16) is to minimize the inventory cost at facilities, transportation cost for the last minute orders, and the penalty cost for shortage or surplus at each retailer's end. In this paper, we derive linear programming equivalent for the second stage CMIP using scenario-based conic MIR cuts and perform computational experiments which demonstrate that these cuts significantly reduce time taken to solve the MM-SFLP-S instances.

We also consider distributionally robust variant of the MM-SFLP-S which is denoted by MM-DRFLP-S and defined as follows:

$$
\min \sum_{i \in P} \sum_{k=1}^{n} c_{i}^{k} x_{i k}+\sum_{i \in P} \sum_{j \in P^{\prime}} t_{i j} z_{i j}+\sum_{i \in P} g_{i} u_{i}+\max _{\left\{\bar{p}_{\omega}\right\}_{\omega \in \Omega} \in \mathfrak{P}} \sum_{\omega \in \Omega} \bar{p}_{\omega} Q_{\omega}(x, z, u)
$$

where $(x, u, z) \in \mathcal{X}, Q_{\omega}(x, z, u)$ is defined by (16)-(18). We utilize the scenario-based CMIR cuts in solving the extensive formulation of the MM-DRFLP-S with finite $|\mathfrak{P}|$ and observe that these cuts are computationally effective.

\subsection{Organization of this paper}

In Sect. 2, we review literature related to TSS-CMIP, TSDR-CMIP, and deterministic CMIP. In Sect. 3, we first review the conic MIR cut [3], and then provide conditions under which addition of cuts derived using conic MIR for the aforementioned deterministic polyhedral conic mixed integer sets is sufficient to provide convex hull or partial convex hull of these sets. In Sect. 4, we provide a reformulation of the second stage CMIP using additional continuous variables, and describe feasible region of the reformulated second stage problem (exactly/approximately) as the intersection of convex hull of a polyhedral conic mixed integer set and a set of $p$-order cones. In Sect. 5, we present conditions under which the scenario-based nonlinear cuts provide partial convex hulls for the feasible set of the deterministic equivalent 
of TSS-CMIPs. To provide special cases of TSS-CMIPs and cutting planes which satisfy these conditions, in Sect. 6, we introduce TSS-CMIPs (and TSDR-CMIPs) with structured CMIPs in the second stage, derive scenario-based cuts for them using conic MIR, and prove that these cuts provide conic/linear programming equivalent or approximation for the second-stage CMIPs. We also demonstrate the applicability of these results for solving the MM-SFLP-S and MM-DRFLP-S. In Sect. 7, we explore the computational effectiveness of the scenariobased cuts by solving instances of MM-SFLP-S, and MM-DRFLP-S, and randomly generated structured TSS-CMIPs and TSDR-CMIPs. We provide concluding remarks in Sect. 8.

\section{Literature review}

We review literature on special cases of TSS-CMIP and TSDR-CMIP in Sect. 2.1 and on cutting planes for deterministic CMIPs in Sect. 2.2.

\subsection{Literature review on special cases of TSS-CMIP and TSDR-CMIP}

One of the most extensively studied special cases of TSS-CMIP is the class of two-stage stochastic mixed integer linear programs (TSS-MILPs), i.e., (1) with $J=\emptyset$ (refer to [33] for a comprehensive survey on TSS-MILPs), which includes the problems whose second stage are pure integer programs [1,31,54], mixed binary programs [19,24,34,44,55,57], or mixed integer programs $[9,55,56,58]$. To solve TSS-MILP, many researchers have been using globally valid linear scenario-based cuts in $\left(x, y_{\omega}\right)$ space, for each scenario $\omega \in \Omega$, to tighten the second stage problems with binary or integer variables, which are then embedded within Benders' decomposition algorithm [12] to solve TSS-MILP. These cuts are of the form $\gamma_{\omega} y_{\omega} \geq \gamma_{\omega, 0}-\gamma_{\omega, 1} x$, where $x$ is a first-stage feasible solution, and $\gamma_{\omega}, \gamma_{\omega, 0}$, and $\gamma_{\omega, 1}$ are realvectors, and are referred to as the "scenario-based" or "parametric" (linear) cuts. For instance, Sherali and Fraticelli [57] derive parametric linear cuts using the reformulation-linearization technique to solve TSS-MILP with $|\Omega|=1$, only binary variables in the first-stage, and mixed binary programs in the second stage. Likewise, Gade et al. [24] utilize parametric Gomory fractional cuts for solving TSS-MILPs with only binary variables in the first stage and nonnegative integer variables in the second stage. Similarly, Bodur et al. [15] use parametric cuts based on split disjunctions to solve TSS-MILP with mixed integer first stage and continuous second stage variables.

In the aforementioned studies, the parametric cuts are developed/added sequentially in the algorithms. Recently, Kim and Mehrotra [30] formulate an integrated staffing and scheduling problem under demand uncertainty as a TSS-MILP with the second stage MIPs having a certain structure and utilize parametric mixed integer rounding inequalities (added a priori) to obtain a linear programming equivalent of the second stage MIPs. Bansal et al. [9] generalize their observation to the general TSS-MILPs and show that under suitable conditions, the second stage MIPs can be convexified by adding parametric cuts a priori. As special cases, the authors consider structured parameterized mixed integer sets or convex objective integer program (COIP) in the second stage. In particular, they extend the results of Miller and Wolsey [39] for deterministic mixed integer sets and COIP to the two-stage stochastic framework, and consider TSS-MILPs with the parametrized version of two special cases of the continuous multi-mixing set $[6,7]$ in the second stage. In this paper, we further generalize their results for TSS-CMIPs, which is equivalent to TSS-MILPs with parametric $p$-order conic constraints in 
the second stage. We utilize parametric cutting planes to obtain a conic/linear programming equivalent or approximation for some structured second stage CMIPs in TSS-CMIPs.

Another special case of TSS-CMIP is two-stage stochastic convex quadratic integer program (TSS-QIP). Özaltin et al. [45] study TSS-QIPs with only stochastic right-hand sides in the second stage along with deterministic quadratic objective functions, linear constraints, and only integer variables in both stages. They reformulate this problem using value functions for quadratic integer programs, and present a global branch-and-bound algorithm and a level-set approach to solve the problem. Mijangos [38] present a branch-and-fix coordination based algorithm to solve TSS-QIP with quadratic terms in the second stage objective function and two-stage stochastic convex problems where the objective function and constraints are nonlinear; both of these problems have binary and continuous variables in the first stage and only continuous variables in the second stage.

The TSDR-CMIPs generalize the two-stage distributionally robust mixed binary programs with general ambiguity set, studied by [8], where both stages have linear constraints and integer variables are bounded between 0 and 1 . Another special case of TSDR-CMIPs are TSDR linear programs where $|J|=0$ and both stages have continuous variables $[8,16,17,50]$. In literature, the ambiguity sets are defined using: linear constraints on the first two moments of the distribution $[13,23,48,53]$, conic constraints to describe the set of distributions with moments [14,22], Kantorovich distance or Wasserstein metric [37,46,47,62], $\zeta$-structure metrics [64], and $\chi^{2}$ distance and Kullback-Leibler divergence [11,18,26,35,61,63]. Recently, Luo and Mehrotra [36] propose a decomposition algorithm to solve general TSDR-CMIP, where the first stage variables are pure binary and the second stage has mixed integer variables. They use branch-and-cut algorithm to solve second stage CMIPs, and generate optimality cuts using disjunctive programming techniques and distribution separation algorithm [8]. In contrast, we consider TSDR-CMIPs with pure integer variables in the first stage and provide conic/linear programming equivalent or approximation for structured CMIPs in the second stage.

\subsection{Brief literature review on CMIPs}

In the last two decades, researchers have extended various classes of cutting planes derived for mixed integer linear programs (MILPs) to mixed integer nonlinear programs (MINLP). It includes extensions of Gomory mixed integer cuts, MIR cuts, split cuts [21], and $n$-step MIR inequalities [28] for MIPs to solve MINLPs [20], second-order CMIPs [3], secondorder conic mixed integer sets [41], and polyhedral CMIPs [52], respectively. Additionally, attempts have been made to derive the convex hull description of (structured) conic mixed integer sets (see $[3,25,29,40]$ for few examples). In this paper, we consider TSS-CMIPs and TSDR-CMIPs with (structured) $p$-order CMIPs in the second stage. Among all papers on deterministic CMIPs, the work in [3] is most closely related to our work. As mentioned before, Atamtürk and Narayanan [3] generalize the MIR inequalities [43] by studying a conic mixed integer set defined by a single second-order conic constraint and one integer variable. They introduce conic MIR cuts which are non-linear in the original space and linear in higher dimensional space. Vinal and Krokhmal [60] extend the conic MIR [3] and lifted conic MIR cuts [4] for second-order CMIPs to derive valid inequalities for $p$-order CMIPs. In this paper, we introduce TSS-CMIPs with multi-constraint $p$-order conic mixed integer sets having multiple integer variables in the second stage and describe the convex hull or tighter approximation of these sets using parametric conic MIR inequalities. 


\section{3 (Partial) convex hull for deterministic polyhedral conic mixed integer sets}

In this section, we first briefly review the conic MIR cut generation procedure [3] which we will use in the proofs of the subsequent theorems. Atamtürk and Narayanan [3] study a single-constraint conic mixed integer set with one integer variable, i.e., $\bar{Z}:=\left\{\left(\sigma, v, \rho_{0}\right) \in\right.$ $\left.\mathbb{Z} \times \mathbb{R}_{+}^{2}: \sqrt{(\sigma-\beta)^{2}+v^{2}} \leq \rho_{0}\right\}$, where $\beta \in \mathbb{R}$. They reformulate set $\bar{Z}$ by using additional continuous variables to get

$$
Z:=\left\{\left(\sigma, v, \rho_{0}, \rho_{1}, \rho_{2}\right) \in \mathbb{Z} \times \mathbb{R}_{+}^{4}:|\sigma-\beta| \leq \rho_{1},|v| \leq \rho_{2}, \sqrt{\rho_{1}^{2}+\rho_{2}^{2}} \leq \rho_{0}\right\} .
$$

Proposition 1 ( [3]) The conic MIR inequality

$$
\left(1-2 \beta^{(1)}\right)(\sigma-\lfloor\beta\rfloor)+\beta^{(1)} \leq \rho_{1}
$$

where $\beta^{(1)}=\beta-\lfloor\beta\rfloor$, is a facet defining inequality for $Z^{1,1}$.

Proposition 2 ( [3]) The convex hull of $\bar{Z}$ is obtained by adding

$$
\sqrt{\left(\left(1-2 \beta^{(1)}\right)(\sigma-\lfloor\beta\rfloor)+\beta^{(1)}\right)^{2}+v^{2}} \leq \rho_{0}
$$

to the continuous relaxation of $\bar{Z}$.

Let $R_{K}^{m}:=\left\{\left(\sigma, \rho, \rho_{0}\right) \in \mathbb{Z}^{K} \times \mathbb{R}_{+}^{m K} \times \mathbb{R}_{+}^{K}: \mathcal{A} \sigma \geq b,\left|\sigma_{k}-\beta_{i k}\right| \leq \rho_{i}^{k}, i=1, \ldots, m, k=\right.$ $1, \ldots, K\}$ and

$$
\begin{aligned}
& T_{K}^{m, u}:=\left\{\left(\eta, \sigma, \rho, \rho_{0}\right) \in \mathbb{Z}^{u} \times \mathbb{Z}^{K} \times \mathbb{R}_{+}^{m K} \times \mathbb{R}_{+}^{K}: \mathcal{A}_{1} \eta+\mathcal{A} \sigma \geq b,\right. \\
&\left.\left\|\rho^{k}\right\|_{1} \leq \rho_{0}^{k},\left|\sum_{t=1}^{u} c_{k t}^{i} \eta_{t}+\sigma_{k}-\beta_{i k}\right| \leq \rho_{i}^{k}, i=1, \ldots, m, k=1, \ldots, K\right\},
\end{aligned}
$$

where $\beta \in \mathbb{R}^{m K}$ and $\mathcal{A} \in \mathbb{R}^{m_{3} \times K}$, be special cases of $S^{m, n}$ and $U_{K}^{m, n, u}$, respectively, with $n=1$ and $g_{k 1}^{i}=1$ for all $(i, k)$. Using the conic MIR cut generation procedure on each defining conic inequality of the sets $R_{K}^{m}, S^{m, n}, T_{K}^{m, u}$, and $U_{K}^{m, n, u}$, in Theorems 1,2 , and 3, we provide (partial) convex hull description of these sets, under certain conditions. The proofs of these theorems are provided in "Appendix D". Note that $Z^{1,1}$ is equivalent to $R_{1}^{1}, S_{1}^{1,1}$, $T_{1}^{1,0}$, and $U_{1}^{1,1,0}$ where $\mathcal{A}$ and $b$ are zero vectors, and $g_{11}^{1}=1$. We denote the fractional part of $\beta_{i k}$ by $\beta_{i k}^{(1)}:=\beta_{i k}-\left\lfloor\beta_{i k}\right\rfloor$.

Motivation behind studying these structured CMIPs comes from the work of Miller and Wolsey [39] who introduced various structured mixed integer linear sets and utilized MIR inequalities of Nemhauser and Wolsey [43] to provide convex hull of these sets. Their results led to a new direction of research in which polyhedral structure of more generalized mixed integer sets have been studied, thereby resulting in new cut-generation approaches for MILPs (see [5-7] for details). Moreover, these sets arise as substructure in the mixed integer programming formulations of variety of applied problems such as production plannning, facility location, and network design problems. Likewise, we extend the results of [3] for conic mixed integer sets with a single constraint and one integer variable and consider structured conic mixed integer sets that have not been studied in the literature. Our results will provide a stepping stone for future polyhedral studies on more generalized conic mixed integer sets, thereby leading to new cut generation approaches for general CMIPs. 
First, we consider a multi-constraint generalization of the simple polyhedral conic mixed integer set $Z_{1,1}$, denoted by $R^{m, k}:=\left\{\left(\sigma_{k}, \rho^{k}\right) \in \mathbb{Z} \times \mathbb{R}^{m}:\left|\sigma_{k}-\beta_{i k}\right| \leq \rho_{i}^{k}, i=1, \ldots, m\right\}$ for $k \in\{1, \ldots, K\}$. Set $R^{m, k}$ has $m$ polyhedral conic constraints with one nonnegative continuous variable in each constraint and an integer variable that is common among all these constraints. In Theorem 1, we derive convex hull of $R_{K}^{m}:=\cap_{k=1}^{K} R^{m, k} \cap\{\sigma: \mathcal{A} \sigma \geq b\}$ using conic MIR cuts.

Theorem 1 If $\mathcal{A}$ is a totally unimodular (TU) matrix and $b$ is integral, then the convex hull of the set $R_{K}^{m}$ is given by

$$
\begin{aligned}
& \left\{\left(\sigma, \rho, \rho_{0}\right) \in \mathbb{R}^{K} \times \mathbb{R}_{+}^{m K} \times \mathbb{R}_{+}^{K}:\right. \\
& \mathcal{A} \sigma \geq b,\left|\sigma_{k}-\beta_{i k}\right| \leq \rho_{i}^{k}, i=1, \ldots, m, k=1, \ldots, K, \\
& \left.\quad\left(1-2 \beta_{i k}^{(1)}\right)\left(\sigma_{k}-\left\lfloor\beta_{i k}\right\rfloor\right)+\beta_{i k}^{(1)} \leq \rho_{i}^{k}, i=1, \ldots, m, k=1, \ldots, K\right\} .
\end{aligned}
$$

Next, in Theorem 2, we consider a multi-integer generalization of the sets $R^{m, k}$ and $R_{K}^{m}$ where each polyhedral conic constraint has $n$ integer variables. We denote this set by $S_{K}^{m, n}$, and note that for $n=1, S_{K}^{m, n}$ reduces to $R_{K}^{m}$. We provide conditions under which addition of conic MIR cuts is sufficient to describe the convex hull of $S_{K}^{m, n}$. In addition, we utilize the foregoing results to demonstrate that under the same conditions, the conic MIR cuts also provide a partial convex hull of a generalization of set $S_{K}^{m, n}$, i.e., $U_{K}^{m, n, u}$, that has $n+u$ number of integer variables in each conic constraint.

Theorem 2 If either of the following conditions is satisfied, i.e.,

(i) $\mathcal{A}$ and $\mathcal{G}^{k}=\left(g_{k t}^{i}\right)$ for $k=1, \ldots, K$, are network flow matrices,

(2) $\mathcal{A}$ is a zero matrix and $\mathcal{G}^{k}=\left(g_{k t}^{i}\right)$ for $k=1, \ldots, K$, are $T U$ matrices,

(3) $\mathcal{A}$ is an identity matrix and $\mathcal{G}^{k}=\left(g_{k t}^{i}\right)$ for $k=1, \ldots, K$, are TU matrices,

and $b$ is integral, then the following results hold: (a) the convex hull of the set $S_{K}^{m, n}$ is obtained by adding inequalities,

$$
\left(1-2 \beta_{i k}^{(1)}\right)\left(\sum_{t=1}^{n} g_{k t}^{i} \sigma_{k t}-\left\lfloor\beta_{i k}\right\rfloor\right)+\beta_{i k}^{(1)} \leq \rho_{i}^{k}, i=1, \ldots, m, k=1, \ldots, K,
$$

to the continuous relaxation of $S_{K}^{m, n}$; and (b) a partial convex hull of the set $U_{K}^{m, n, u}$ is given by

$$
\begin{aligned}
& U_{K, p c h}^{m, n, u}:=\left\{\left(\eta, \sigma, \rho, \rho_{0}\right) \in \mathbb{Z}^{u} \times \mathbb{R}^{n K} \times \mathbb{R}_{+}^{m K} \times \mathbb{R}_{+}^{K}: \mathcal{A}_{1} \eta+\mathcal{A} \sigma \geq b,\right. \\
& \left\|\rho^{k}\right\|_{1} \leq \rho_{0}^{k}, \quad\left|\sum_{t=1}^{u} c_{k t}^{i} \eta_{t}+\sum_{t=1}^{n} g_{k t}^{i} \sigma_{k t}-\beta_{i k}\right| \leq \rho_{i}^{k}, i=1, \ldots, m, k=1, \ldots, K, \\
& \left.\left(1-2 \beta_{i k}^{(1)}\right)\left(\sum_{t=1}^{u} c_{k t}^{i} \eta_{t}+\sum_{t=1}^{n} g_{k t}^{i} \sigma_{k t}-\left\lfloor\beta_{i k}\right\rfloor\right)+\beta_{i k}^{(1)} \leq \rho_{i}^{k}, i=1, \ldots, m, k=1, \ldots, K\right\} .
\end{aligned}
$$

In Theorem 3, we derive a partial convex hull of the set $U_{K}^{m, n, u}$ for $n=1$, denoted by $T_{K}^{m, u}$, using conic MIR cuts under the condition that matrix $\mathcal{A}$ is TU (which is not considered in Theorem 2). 
Theorem 3 If $\mathcal{A}$ is a TU matrix and $b$ is integral, then a partial convex hull of the set $T_{K}^{m, u}$ is given by

$$
\begin{aligned}
& T_{K, p c h}^{m, u}:=\left\{\left(\eta, \sigma, \rho, \rho_{0}\right) \in \mathbb{Z}^{u} \times \mathbb{R}^{K} \times \mathbb{R}_{+}^{m K} \times \mathbb{R}_{+}^{K}: \mathcal{A}_{1} \eta+\mathcal{A} \sigma \geq b,\right. \\
& \left\|\rho^{k}\right\|_{1} \leq \rho_{0}^{k}, \quad\left|\sum_{t=1}^{u} c_{k t}^{i} \eta_{t}+\sigma_{k}-\beta_{i k}\right| \leq \rho_{i}^{k}, i=1, \ldots, m, k=1, \ldots, K, \\
& \left.\left(1-2 \beta_{i k}^{(1)}\right)\left(\sum_{t=1}^{u} c_{k t}^{i} \eta_{t}+\sigma_{k}-\left\lfloor\beta_{i k}\right\rfloor\right)+\beta_{i k}^{(1)} \leq \rho_{i}^{k}, i=1, \ldots, m, k=1, \ldots, K\right\} .
\end{aligned}
$$

\section{Reformulation of second stage of TSS-CMIPs and TSDR-CMIPs}

We reformulate the second stage problem $\overline{\mathcal{Q}}_{\omega}(x)$ of the TSS-CMIP and TSDR-CMIP using additional continuous variables, as follows:

$$
\begin{aligned}
\mathcal{Q}_{\omega}(x):=\min \quad & g_{\omega} y_{\omega}+\sum_{j \in J} \hat{g}_{\omega}^{j} d_{\omega, 0}^{j} \\
\text { s.t. } \quad & W_{\omega} y_{\omega} \geq r_{\omega}-T_{\omega} x, \\
& \left|e_{\omega, i}^{j} y_{\omega}^{j}+f_{\omega, i}^{j} x-h_{\omega, i}^{j}\right| \leq d_{\omega, i}^{j}, q u a d i=1, \ldots, m_{2}, j \in J, \\
& \left\|d_{\omega}^{j}\right\|_{p} \leq d_{\omega, 0}^{j}, \quad j \in J, \\
& y_{\omega}^{j} \in \mathbb{Z}^{q}, d_{\omega, i}^{j} \in \mathbb{R}_{+}, \quad i=0,1, \ldots, m_{2}, j \in J,
\end{aligned}
$$

where $d_{\omega}^{j}:=\left(d_{\omega, 1}^{j}, \ldots, d_{\omega, m_{2}}^{j}\right) \in \mathbb{R}_{+}^{m_{2}}$ for $j \in J, e_{\omega, i}^{j}$ and $f_{\omega, i}^{j}$ denote the $i$ th row of matrices $E_{\omega}^{j}$ and $F_{\omega}^{j}$, respectively, and $h_{\omega, i}^{j}$ denotes the $i$ th element of real vector $h_{\omega}^{j}$. We define the feasible region of the reformulated second stage program by $\mathcal{K}_{\omega}(x):=\left\{\left(y_{\omega}, d_{\omega}\right)\right.$ : (22)-(25) hold\} for $x \in X$ and $\omega \in \Omega$. We note that $\overline{\mathcal{K}}_{\omega}(x)$ is in lower dimensional space by rewriting (23) and (24).

Next, we describe the feasible region of the reformulated second stage problem (exactly/approximately) as intersection of convex hull of a polyhedral conic mixed integer set and a set of $p$-order cones. Let $\mathcal{K}_{\omega}(x)=\mathcal{K}_{\omega}^{1}(x) \cap \mathcal{K}_{\omega}^{2}(x)$ where $\mathcal{K}_{\omega}^{1}(x):=$ $\left\{\left(y_{\omega}, d_{\omega}\right) \in \mathbb{Z}^{q|J|} \times \mathbb{R}_{+}^{m_{2}|J|+|J|}:(22)-(23)\right.$ hold $\}$ is a polyhedral conic mixed integer set and $\mathcal{K}_{\omega}^{2}(x):=\left\{\left(y_{\omega}, d_{\omega}\right) \in \mathbb{R}^{q|J|} \times \mathbb{R}_{+}^{m_{2}|J|+|J|}:(24)\right.$ holds $\}$ is an intersection of $|J|$ number of $p$-order cones. In Theorem 4 , we provide a relation between the convex hull of $\mathcal{K}_{\omega}(x)$ and the convex hull of $\mathcal{K}_{\omega}^{1}(x)$.

Theorem 4 For each $x \in X$ and $\omega \in \Omega$, if $p=1$,

$$
\operatorname{conv}\left(\mathcal{K}_{\omega}(x)\right)=\operatorname{conv}\left(\mathcal{K}_{\omega}^{1}(x)\right) \cap \mathcal{K}_{\omega}^{2}(x),
$$

and if $p \geq 2, \operatorname{conv}\left(\mathcal{K}_{\omega}(x)\right) \subseteq \operatorname{conv}\left(\mathcal{K}_{\omega}^{1}(x)\right) \cap \mathcal{K}_{\omega}^{2}(x)$.

Proof For $x \in X$ and $\omega \in \Omega, \mathcal{K}_{\omega}(x)=\mathcal{K}_{\omega}^{1}(x) \cap \mathcal{K}_{\omega}^{2}(x)$. Therefore,

$$
\operatorname{conv}\left(\mathcal{K}_{\omega}(x)\right) \subseteq \operatorname{conv}\left(\mathcal{K}_{\omega}^{1}(x)\right) \cap \mathcal{K}_{\omega}^{2}(x) .
$$


Now, for $p=1$, assume that a point $\hat{\eta}_{\omega}=\left(\hat{y}_{\omega},\left\{\hat{d}_{\omega}^{j}, \hat{d}_{\omega, 0}^{j}\right\}_{j \in J}\right)$ belongs to $\operatorname{conv}\left(\mathcal{K}_{\omega}^{1}(x)\right) \cap \mathcal{K}_{\omega}^{2}(x)$, i.e. $\hat{\eta}_{\omega} \in \operatorname{conv}\left(\mathcal{K}_{\omega}^{1}(x)\right)$ and $\hat{\eta}_{\omega} \in \mathcal{K}_{\omega}^{2}(x)$ or $\hat{d}_{\omega, 0}^{j}-\sum_{i=1}^{m_{2}} \hat{d}_{\omega, i}^{j} \geq 0$. Since $\hat{\eta}_{\omega} \in \operatorname{conv}\left(\mathcal{K}_{\omega}^{1}(x)\right), \hat{\eta}_{\omega}$ can be written as convex combination of a finite number of points $\bar{\eta}_{\omega}^{k}=\left(\bar{y}_{\omega}^{k},\left\{\bar{d}_{\omega}^{j, k}, \bar{d}_{\omega, 0}^{j, k}\right\}_{j \in J}\right) \in \mathcal{K}_{\omega}^{1}(x)$, where we define $\bar{d}_{\omega, 0}^{j, k}=\hat{d}_{\omega, 0}^{j}+$ $\sum_{i=1}^{m_{2}}\left(\bar{d}_{\omega, i}^{j, k}-\hat{d}_{\omega, i}^{j}\right)$ for $j \in J$ and $k \in\left\{1,2, \ldots, q|J|+1+\left(m_{2}+1\right)|J|\right\}$, i.e., for $\lambda_{k} \in[0,1]$ and $\sum_{k} \lambda_{k}=1, \sum_{k} \lambda_{k} \bar{\eta}_{\omega}^{k}=\hat{\eta}_{\omega}$. Observe that for all $(j, k), \bar{d}_{\omega, 0}^{j, k} \geq 0$ as $\hat{d}_{\omega, 0}^{j}-\sum_{i=1}^{m_{2}} \hat{d}_{\omega, i}^{j} \geq 0$ and $\bar{d}_{\omega, i}^{j, k} \geq 0$ for all $i=1, \ldots, m_{2}$. Also, note that $\bar{\eta}_{\omega}^{k} \in \mathcal{K}_{\omega}^{2}(x)$ as $\bar{d}_{\omega, 0}^{j, k}=\hat{d}_{\omega, 0}^{j}+\sum_{i=1}^{m_{2}}\left(\bar{d}_{\omega, i}^{j, k}-\hat{d}_{\omega, i}^{j}\right) \geq \sum_{i=1}^{m_{2}} \bar{d}_{\omega, i}^{j, k}$. Hence, $\bar{\eta}_{\omega}^{k} \in \mathcal{K}_{\omega}^{1}(x) \cap \mathcal{K}_{\omega}^{2}(x)$ for all $k$. This implies $\sum_{k} \lambda_{k} \bar{\eta}_{\omega}^{k}=\hat{\eta}_{\omega} \in \operatorname{conv}\left(\mathcal{K}_{\omega}^{1}(x) \cap \mathcal{K}_{\omega}^{2}(x)\right)=\operatorname{conv}\left(\mathcal{K}_{\omega}(x)\right)$, and therefore, $\operatorname{conv}\left(\mathcal{K}_{\omega}^{1}(x)\right) \cap \mathcal{K}_{\omega}^{2}(x) \subseteq \operatorname{conv}\left(\mathcal{K}_{\omega}(x)\right)$. Hence, for $p=1$, we get

$$
\operatorname{conv}\left(\mathcal{K}_{\omega}(x)\right)=\operatorname{conv}\left(\mathcal{K}_{\omega}^{1}(x)\right) \cap \mathcal{K}_{\omega}^{2}(x) .
$$

and this completes the proof.

\section{Scenario-based cuts for extensive formulation of TSS-CMIPs}

In this section, we present sufficient conditions under which the integrality restrictions on the second stage integer variables of the TSS-CMIPs can be relaxed (without impacting the integrality of the optimal solution) by adding scenario-based nonlinear inequalities in $\left(x, y_{\omega}, d_{\omega}\right)$ space to the extensive formulation of TSS-CMIPs. In other words, using these scenario-based cuts, we derive "partial convex hull(s)" for $\overline{\mathcal{P}}$, which is the feasible region of the deterministic equivalent of TSS-CMIPs. Given a nonempty set $\Gamma \subseteq \Omega$, we define a partial convex hull of $\overline{\mathcal{P}}$ by another conic mixed integer set,

$$
\begin{array}{cc}
\overline{\mathcal{P}}_{p c h}:=\left\{T_{\omega} x+W_{\omega} y_{\omega} \geq r_{\omega},\right. & \omega \in \Omega, \\
\left\|E_{\omega}^{j} y_{\omega}^{j}+F_{\omega}^{j} x-h_{\omega}^{j}\right\|_{p} \leq d_{\omega, 0}^{j}, & j \in J, \omega \in \Omega, \\
\left\|\bar{E}_{\omega, l}^{j} y_{\omega}^{j}+\bar{F}_{\omega, l}^{j} x-\bar{h}_{\omega, l}^{j}\right\|_{p} \leq d_{\omega, 0}^{j}, & l \in \mathcal{L}, j \in J, \omega \in \Gamma, \\
x \in X, d \in \mathbb{R}_{+}^{|J| \times|\Omega|}, y_{\omega}^{j} \in \mathbb{R}^{q}, & j \in J, \omega \in \Gamma, \\
y_{\omega}^{j} \in \mathbb{Z}^{q}, & j \in J, \omega \in \Omega \backslash \Gamma\},
\end{array}
$$

where for each $l \in \mathcal{L}, j \in J$, and $\omega \in \Gamma, \bar{E}_{\omega, l}^{j}, \bar{F}_{\omega, l}^{j}, \bar{h}_{\omega, l}^{j}$ are matrices (or vectors) corresponding to scenario-based cuts in $\left(x, y_{\omega}, d_{\omega}\right)$ space added a priori to $\overline{\mathcal{P}}$, such that $\overline{\mathcal{P}} \subseteq \overline{\mathcal{P}}_{p c h} \subseteq \operatorname{conv}(\overline{\mathcal{P}})=\operatorname{conv}\left(\overline{\mathcal{P}}_{p c h}\right)$. Note that $\overline{\mathcal{P}}_{p c h}$ has fewer number of integrality constraints (but possibly more linear or nonlinear inequalities) than $\overline{\mathcal{P}}$. Whereas in comparison to $\operatorname{conv}(\mathcal{P}), \mathcal{P}_{\text {pch }}$ might have lesser inequalities but more integrality constraints.

Theorem 5 Given a nonempty set $\Gamma \subseteq \Omega$, if conv $\left(\overline{\mathcal{K}}_{\omega}(x)\right)=\overline{\mathcal{K}}_{\text {tight }}^{\omega}(x)$ for all $x \in X$ and $\omega \in \Gamma$, where

$$
\overline{\mathcal{K}}_{\text {tight }}^{\omega}(x):=\left\{\left(y_{\omega}, d_{\omega}\right) \in \mathbb{R}^{q|J|} \times \mathbb{R}_{+}^{|J|}: W_{\omega} y_{\omega} \geq r_{\omega}-T_{\omega} x,\right.
$$




$$
\begin{aligned}
& \left\|E_{\omega}^{j} y_{\omega}^{j}+F_{\omega}^{j} x-h_{\omega}^{j}\right\|_{p} \leq d_{\omega, 0}^{j}, j \in J, \\
& \left.\left\|\bar{E}_{\omega, l}^{j} y_{\omega}^{j}+\bar{F}_{\omega, l}^{j} x-\bar{h}_{\omega, l}^{j}\right\|_{p} \leq d_{\omega, 0}^{j}, l \in \mathcal{L}, j \in J\right\},
\end{aligned}
$$

then $\overline{\mathcal{P}}_{\text {pch }}$ is a partial convex hull of $\overline{\mathcal{P}}$, i.e.,

$\overline{\mathcal{P}} \subseteq \overline{\mathcal{P}}_{p c h} \subseteq \operatorname{conv}(\overline{\mathcal{P}})=\operatorname{conv}\left(\overline{\mathcal{P}}_{p c h}\right)$.

Proof Refer to "Appendix A".

Remark 1 Theorem 5 for TSS-CMIP extends the results (Lemma 1 and Theorem 3) of [9] for TSS-MILP, i.e., TSS-CMIP with $|J|=0$, with linear parametric cuts. In Sect. 6, we introduce structured TSS-CMIPs and derive classes of cutting planes for them which satisfy the conditions stated in Theorem 5.

\section{Structured two-stage stochastic and distributionally robust $p$-order conic mixed integer programs}

In this section, we introduce TSS-CMIPs and TSDR-CMIPs with structured $p$-order CMIPs in the second stage, derive scenario-based cuts using conic MIR for them, and prove that these cuts along with the defining constraints provide conic/linear programming equivalent or approximation for the second stage CMIPs. We also demonstrate the applicability of these results for solving MM-SFLP-S. Specifically, we consider the following three structured CMIPs in the second stage, i.e. $\overline{\mathcal{Q}}_{\omega}(x)$ (or $\mathcal{Q}_{\omega}(x)$ ) where

(a) $E_{\omega}=\mathbf{I}_{2}, F_{\omega}^{j}=[1,0]^{T}$ for $j \in J$, and $m_{3}=0$ (Corollary 1$)$;

(b) $E_{\omega}^{j}=\mathbf{1}$ for $j \in J$ and $W_{\omega}$ is TU (Theorem 6);

(c) $E_{\omega}^{j}$ for all $j \in J$, and $W_{\omega}$ are network flow matrices (Theorem 7(i));

(d) $E_{\omega}^{j}$ for all $j \in J$ are TU matrices, and $W_{\omega}=\mathbf{I}_{m_{3}}$ or $W_{\omega}=\mathbf{0}$ (Theorem 7(ii)).

\subsection{Tight second stage formulations for structured TSS-CMIPs and TSDR-CMIPs}

We derive classes of parametric (non)-linear inequalities using conic MIR to get conic/linear programming equivalent or approximation for the second stage problems. It is important to note that for structured TSS-CMIP (a), we utilize the result of [3] for $\bar{Z}$ in Corollary 1; whereas for structures (b), (c), and (d), no result is known for multi-constraint and multivariable generalizations of $\bar{Z}$ or $Z_{1}$, except Theorems $1-3$. We introduce case (a) mainly to present a simple example that illustrates how parametric nonlinear inequalities can be used to get conic programming equivalent of the second stage CMIP of TSS-CMIPs and TSDR-CMIPs.

Corollary 1 In TSS-CMIP (1) and TSDR-CMIP (9), let

$$
\begin{aligned}
& \overline{\mathcal{Q}}_{\omega}(x):= \min g_{\omega}^{1} y_{\omega, 1}+g_{\omega}^{2} y_{\omega, 2}+\hat{g}_{\omega} d_{\omega, 0} \\
& \text { s.t. } \sqrt{\left(y_{\omega, 1}+f_{\omega} x-h_{\omega}\right)^{2}+\left(y_{\omega, 2}\right)^{2}} \leq d_{\omega, 0}, \\
& y_{\omega, 1} \in \mathbb{Z}, y_{\omega, 2} \in \mathbb{R}_{+}, d_{\omega, 0} \in \mathbb{R}_{+} .
\end{aligned}
$$

The convex hull of the feasible region of $\overline{\mathcal{Q}}_{\omega}(x)$ for all $x \in X$ is given by

$$
\left\{\left(y_{\omega, 1}, y_{\omega, 2}, d_{\omega, 0}\right) \in \mathbb{R} \times \mathbb{R}_{+}^{2}:\right. \text { (30) and }
$$




$$
\left.\sqrt{\left(\left(1-2 \mu_{\omega}\right)\left(y_{\omega, 1}+f_{\omega} x-h_{\omega}\right)+\mu_{\omega}\right)^{2}+\left(y_{\omega, 2}\right)^{2}} \leq d_{\omega, 0}\right\},
$$

where $\mu_{\omega}=h_{\omega}-\left\lfloor h_{\omega}\right\rfloor$. Furthermore, in higher dimensional space $\overline{\mathcal{Q}}_{\omega}(x)$ can be reformulated as:

$$
\begin{aligned}
& \mathcal{Q}_{\omega}(x):= \min \left\{g_{\omega}^{1} y_{\omega, 1}+g_{\omega}^{2} y_{\omega, 2}+\hat{g}_{\omega} d_{\omega, 0}:\left|y_{\omega, 1}+f_{\omega} x-h_{\omega}\right| \leq d_{\omega, 1},\right. \\
&\left|y_{\omega, 2}\right| \leq d_{\omega, 2}, \sqrt{d_{\omega, 1}^{2}+d_{\omega, 2}^{2}} \leq d_{\omega, 0}, \\
&\left.y_{\omega, 1} \in \mathbb{Z}, y_{\omega, 2} \in \mathbb{R}_{+}, d_{\omega, i} \in \mathbb{R}_{+}, i=0,1,2\right\} .
\end{aligned}
$$

Then, for all $x \in X$, the convex hull of the feasible region of $\mathcal{Q}_{\omega}(x)$, denoted by $\mathcal{K}_{\omega}(x)$, is obtained by adding the following parametric linear inequality to the continuous relaxation of $\mathcal{K}_{\omega}(x)$ :

$$
\left(1-2 \mu_{\omega}\right)\left(y_{\omega, 1}-\left\lfloor h_{\omega}\right\rfloor+f_{\omega} x\right)+\mu_{\omega} \leq d_{\omega, 1} .
$$

Proof Refer to "Appendix B.1".

As mentioned before, the motivation behind the ensuing theorems is to extend the results of Bansal et al. [9] for structured TSS-MILPs to structured TSS-CMIPs and TSDR-CMIPs. More specifically, in [9], the authors considered structured mixed integer sets studied by Miller and Wolsey [39] and two special cases of the continuous multi-mixing set [6,7] in the second stage of TSS-MILPs, and provide linear programming equivalent for the second stage programs. We also obtain a conic/linear programming equivalent or approximation for aforementioned structured second stage CMIPs in TSS-CMIPs and TSDR-CMIPs, thereby extending our results for deterministic CMIPs (Theorems 1 and 2 for $R_{K}^{m}$ and $S_{K}^{m, n}$, respectively) to stochastic CMIPs. In Theorem 6, we consider second stage problems with $|J|$ conic constraints (34) where each constraint has only one integer variables $y_{\omega}^{j} \in \mathbb{Z}$ associated to it and these integer variables $y_{\omega}=\left(y_{\omega}^{1}, y_{\omega}^{2}, \ldots, y_{\omega}^{|J|}\right) \in \mathbb{Z}^{|J|}$ are connected with linear constraints (33).

Theorem 6 In TSS-CMIP (1) and TSDR-CMIP (9), let

$$
\begin{aligned}
& \overline{\mathcal{Q}}_{\omega}(x):=\min g_{\omega} y_{\omega}+\sum_{j \in J} \hat{g}_{\omega}^{j} d_{\omega, 0}^{j} \\
& \text { s.t. } W_{\omega} y_{\omega} \geq r_{\omega}-T_{\omega} x \text {, } \\
& \left\|\mathbf{1} y_{\omega}^{j}+F_{\omega}^{j} x-h_{\omega}^{j}\right\|_{p} \leq d_{\omega, 0}^{j}, \quad j \in J, \\
& y_{\omega}^{j} \in \mathbb{Z}, d_{\omega, 0}^{j} \in \mathbb{R}_{+}, \quad j \in J,
\end{aligned}
$$

where $W_{\omega}$ is a TU matrix and $r_{\omega}$ is integral. For $p=1$ or $p \geq 2$, the convex hull or an approximation, respectively, of the feasible region of $\overline{\mathcal{Q}}_{\omega}(x)$ for all $x \in X$ are given by

$$
\begin{aligned}
& \left\{\left(y_{\omega}, d_{\omega, 0}\right) \in \mathbb{R}^{|J|} \times \mathbb{R}_{+}^{|J|}:(33),(34),\right. \text { and } \\
& \left.\left\|\bar{E}_{\omega, l}^{j} y_{\omega}^{j}+\bar{F}_{\omega, l}^{j} x-\bar{h}_{\omega, l}^{j}\right\|_{p} \leq d_{\omega, 0}^{j}, \quad l \in \mathcal{L}, j \in J\right\},
\end{aligned}
$$

where the ith element of $\bar{E}_{\omega, l}^{j} y_{\omega}^{j}+\bar{F}_{\omega, l}^{j} x-\bar{h}_{\omega, l}^{j}$ is either $y_{\omega}^{j}+f_{\omega, i}^{j} x-h_{\omega, i}^{j}$ or $\left(1-2 \mu_{\omega, i}^{j}\right)\left(y_{\omega}^{j}-\left\lfloor h_{\omega, i}^{j}\right\rfloor+f_{\omega, i}^{j} x\right)+\mu_{\omega, i}^{j}$, and $\mu_{\omega, i}^{j}=h_{\omega, i}^{j}-\left\lfloor h_{\omega, i}^{j}\right\rfloor$. 
Furthermore, in higher dimensional space $\overline{\mathcal{Q}}_{\omega}(x)$ can be reformulated as:

$$
\begin{aligned}
\mathcal{Q}_{\omega}(x):= & \min \left\{g_{\omega} y_{\omega}+\sum_{j \in J} \hat{g}_{\omega}^{j} d_{\omega, 0}^{j}:\right. \text { (33), and } \\
& \left|y_{\omega}^{j}+f_{\omega, i}^{j} x-h_{\omega, i}^{j}\right| \leq d_{\omega, i}^{j}, \quad i=1, \ldots, m_{2}, \quad j \in J, \\
& \left\|d_{\omega}^{j}\right\|_{p} \leq d_{\omega, 0}^{j}, \quad j \in J, \\
& \left.y_{\omega}^{j} \in \mathbb{Z}, d_{\omega, 0}^{j} \in \mathbb{R}_{+}, d_{\omega}^{j} \in \mathbb{R}_{+}^{m_{2}}, \quad j \in J\right\} .
\end{aligned}
$$

Then, for all $x \in X$, the convex hull (for $p=1$ ) and an approximation (for $p \geq 2$ ) of the feasible region of $\mathcal{Q}_{\omega}(x)$, denoted by $\mathcal{K}_{\omega}(x)$, are obtained by adding $m_{2} \times|J|$ number of the following parametric linear inequalities (in the higher dimensional space) to the continuous relaxation of $\mathcal{K}_{\omega}(x):\left(1-2 \mu_{\omega, i}^{j}\right)\left(y_{\omega}^{j}-\left\lfloor h_{\omega, i}^{j}\right\rfloor+f_{\omega, i}^{j} x\right)+\mu_{\omega, i}^{j} \leq d_{\omega, i}^{j}, \quad i=$ $1, \ldots, m_{2}, j \in J$.

\section{Proof Let}

$$
\begin{aligned}
\mathcal{K}_{\omega}^{1}(x)= & \left\{\left(y_{\omega}, d_{\omega}\right) \in \mathbb{Z}^{|J|} \times \mathbb{R}_{+}^{m_{2}|J|+|J|}:\right. \\
& W_{\omega} y_{\omega} \geq r_{\omega}-T_{\omega} x, \\
& \left.\left|y_{\omega}^{j}+f_{\omega, i}^{j} x-h_{\omega, i}^{j}\right| \leq d_{\omega, i}^{j}, \quad i=1, \ldots, m_{2}, \quad j \in J\right\}
\end{aligned}
$$

for all $x \in X$. First we apply Proposition 1 to each defining inequality (42) of $\mathcal{K}_{\omega}^{1}(x)$. In other words, we substitute $\sigma=y_{\omega}^{j}, \beta=h_{\omega, i}^{j}-f_{\omega, i}^{j} x$, and $\rho_{1}=d_{\omega, i}^{j}$ in $Z_{1}$ and get the following valid parametric conic MIR inequalities (20) for $\mathcal{K}_{\omega}^{1}(x)$ :

$$
\left(1-2 \mu_{\omega, i}^{j}\right)\left(y_{\omega}^{j}-\left\lfloor h_{\omega, i}^{j}-f_{\omega, i}^{j} x\right\rfloor\right)+\mu_{\omega, i}^{j} \leq d_{\omega, i}^{j}, \quad i=1, \ldots, m_{2}, j \in J .
$$

Since $f_{\omega, i}^{j} x$ is integral for all $x \in X,\left\lfloor f_{\omega, i}^{j} x\right\rfloor=f_{\omega, i}^{j} x$ and therefore, inequality (43) is equivalent to

$$
\left(1-2 \mu_{\omega, i}^{j}\right)\left(y_{\omega}^{j}-\left\lfloor h_{\omega, i}^{j}\right\rfloor+f_{\omega, i}^{j} x\right)+\mu_{\omega, i}^{j} \leq d_{\omega, i}^{j}, \quad i=1, \ldots, m_{2}, j \in J .
$$

Thus,

$$
\operatorname{conv}\left(\mathcal{K}_{\omega}^{1}(x)\right) \subseteq \mathcal{K}_{\omega}^{3}(x):=\left\{\left(y_{\omega}, d_{\omega}\right) \in \mathbb{R}^{|J|} \times \mathbb{R}_{+}^{m_{2}|J|+|J|}: \text { (41), (42), (44) hold }\right\}
$$

for all $x \in X$. Notice that we can rewrite the set $\mathcal{K}_{\omega}^{3}(x)$ as

$$
\begin{aligned}
\mathcal{K}_{\omega}^{3}(x)= & \left\{\left(y_{\omega}, d_{\omega}\right) \in \mathbb{R}^{|J|} \times \mathbb{R}_{+}^{m_{2}|J|+|J|}: W_{\omega} y_{\omega} \geq r_{\omega}-T_{\omega} x,\right. \\
& d_{\omega, i}^{j} \geq y_{\omega}^{j}-\left(h_{\omega, i}^{j}-f_{\omega, i}^{j} x\right), \quad i=1, \ldots, m_{2}, j \in J, \\
& d_{\omega, i}^{j} \geq\left(h_{\omega, i}^{j}-f_{\omega, i}^{j} x\right)-y_{\omega}^{j}, \quad i=1, \ldots, m_{2}, j \in J, \\
d_{\omega, i}^{j} \geq & \left.\left(1-2 \mu_{\omega, i}^{j}\right)\left(y_{\omega}^{j}-\left\lfloor h_{\omega, i}^{j}\right\rfloor+f_{\omega, i}^{j} x\right)+\mu_{\omega, i}^{j}, i=1, \ldots, m_{2}, j \in J\right\} .
\end{aligned}
$$


Now let $\mathcal{K}_{\omega}^{4}(x)$ be a bounded face of $\operatorname{Proj}_{y_{\omega}, d_{1}, \ldots, d_{|\Omega|}} \mathcal{K}_{\omega}^{3}(x)$ with maximum possible dimension. Since $\mathcal{K}_{\omega}^{4}(x)$ is a bounded face, for all points $\left(y_{\omega}, d_{1}, \ldots, d_{|\Omega|}\right) \in \mathcal{K}_{\omega}^{4}(x), d_{\omega, i}^{j} \leq \hat{d}_{\omega, i}^{j}$ for $i=1, \ldots, m_{2}$ and $j \in J$, where $\left(\hat{y}_{\omega}, \hat{d}_{1}, \ldots, \hat{d}_{|\Omega|}\right) \in \operatorname{Proj}_{y_{\omega}, d_{1}, \ldots, d_{|\Omega|} \mid} \mathcal{K}_{\omega}^{3}(x)$. Thus, for $i=1, \ldots, m_{2}$ and $j \in J$, we have

$$
\begin{gathered}
d_{\omega, i}^{j}=\max \left\{y_{\omega}^{j}-\left(h_{\omega, i}^{j}-f_{\omega, i}^{j} x\right),\left(h_{\omega, i}^{j}-f_{\omega, i}^{j} x\right)-y_{\omega}^{j},\right. \\
\left.\left(1-2 \mu_{\omega, i}^{j}\right)\left(y_{\omega}^{j}-\left\lfloor h_{\omega, i}^{j}\right\rfloor+f_{\omega, i}^{j} x\right)+\mu_{\omega, i}^{j}\right\} .
\end{gathered}
$$

Moreover, if $h_{\omega, i}^{j} \in \mathbb{Z}$ for all $j \in J$ then $\mu_{\omega, i}^{j}=0$, and as a result inequality (44) reduces to inequality (46). However, when $h_{\omega, i}^{j} \notin \mathbb{Z}$ for $j \in J$, then there are three possible cases:

Case I. $d_{\omega, i}^{j}=y_{\omega}^{j}-\left(h_{\omega, i}^{j}-f_{\omega, i}^{j} x\right)$ : This case will happen if and only if $y_{\omega}^{j}-$ $\left(h_{\omega, i}^{j}-f_{\omega, i}^{j} x\right) \geq\left(h_{\omega, i}^{j}-f_{\omega, i}^{j} x\right)-y_{\omega}^{j}$ and $y_{\omega}^{j}-\left(h_{\omega, i}^{j}-f_{\omega, i}^{j} x\right) \geq\left(1-2 \mu_{\omega, i}^{j}\right)$ $\left(y_{\omega}^{j}-\left\lfloor h_{\omega, i}^{j}\right\rfloor+f_{\omega, i}^{j} x\right)+\mu_{\omega, i}^{j}$, which are equivalent to $y_{\omega}^{j} \geq h_{\omega, i}^{j}-f_{\omega, i}^{j} x$ and $y_{\omega}^{j} \geq$ $\left\lceil h_{\omega, i}^{j}\right\rceil-f_{\omega, i}^{j} x$, respectively. Note that the last inequality is stronger than the second last inequality. Therefore, we can claim that $d_{\omega, i}^{j}=y_{\omega}^{j}-\left(h_{\omega, i}^{j}-f_{\omega, i}^{j} x\right)$ if and only if $y_{\omega}^{j} \geq\left\lceil h_{\omega, i}^{j}\right\rceil-f_{\omega, i}^{j} x$.

Case II. $d_{\omega, i}^{j}=h_{\omega, i}^{j}-f_{\omega, i}^{j} x-y_{\omega}^{j}$ : This case will happen if and only if $h_{\omega, i}^{j}-f_{\omega, i}^{j} x-y_{\omega}^{j} \geq$ $y_{\omega}^{j}-\left(h_{\omega, i}^{j}-f_{\omega, i}^{j} x\right)$ and $h_{\omega, i}^{j}-f_{\omega, i}^{j} x-y_{\omega}^{j} \geq\left(1-2 \mu_{\omega, i}^{j}\right)$ $\left(y_{\omega}^{j}-\left\lfloor h_{\omega, i}^{j}\right\rfloor+f_{\omega, i}^{j} x\right)+\mu_{\omega, i}^{j}$, which are equivalent to $y_{\omega}^{j} \leq h_{\omega, i}^{j}-f_{\omega, i}^{j} x$ and $y_{\omega}^{j} \leq$ $\left\lfloor h_{\omega, i}^{j}\right\rfloor-f_{\omega, i}^{j} x$, respectively, as $\mu_{\omega, i}^{j} \leq 1$. Again, note that the last inequality is stronger than the second last inequality. Therefore, $d_{\omega, i}^{j}=h_{\omega, i}^{j}-f_{\omega, i}^{j} x-y_{\omega}^{j}$ if and only if $y_{\omega}^{j} \leq\left\lfloor h_{\omega, i}^{j}\right\rfloor-f_{\omega, i}^{j} x$.

Case III. $d_{\omega, i}^{j}=\left(1-2 \mu_{\omega, i}^{j}\right)\left(y_{\omega}^{j}-\left\lfloor h_{\omega, i}^{j}\right\rfloor+f_{\omega, i}^{j} x\right)+\mu_{\omega, i}^{j}$ : This case will happen if and only if $\left(1-2 \mu_{\omega, i}^{j}\right)\left(y_{\omega}^{j}-\left\lfloor h_{\omega, i}^{j}\right\rfloor+f_{\omega, i}^{j} x\right)+\mu_{\omega, i}^{j} \geq h_{\omega, i}^{j}-f_{\omega, i}^{j} x-y_{\omega}^{j}$ and $\left(1-2 \mu_{\omega, i}^{j}\right)\left(y_{\omega}^{j}-\left\lfloor h_{\omega, i}^{j}\right\rfloor+f_{\omega, i}^{j} x\right)+\mu_{\omega, i}^{j} \geq y_{\omega}^{j}-\left(h_{\omega, i}^{j}-f_{\omega, i}^{j} x\right)$, which are equivalent to $y_{\omega}^{j} \geq\left\lfloor h_{\omega, i}^{j}\right\rfloor-f_{\omega, i}^{j} x$ and $y_{\omega}^{j} \leq\left\lceil h_{\omega, i}^{j}\right\rceil-f_{\omega, i}^{j} x$, respectively. Therefore, $d_{\omega, i}^{j}=\left(1-2 \mu_{\omega, i}^{j}\right)\left(y_{\omega}^{j}-\left\lfloor h_{\omega, i}^{j}\right\rfloor+f_{\omega, i}^{j} x\right)+\mu_{\omega, i}^{j}$ if and only if $\left\lfloor h_{\omega, i}^{j}\right\rfloor-f_{\omega, i}^{j} x \leq y_{\omega}^{j} \leq$ $\left\lceil h_{\omega, i}^{j}\right\rceil-f_{\omega, i}^{j} x$.

Next, for each $j \in J$, we partition the set $\mathcal{I}:=\left\{1, \ldots, m_{2}\right\}$ into the sets $\mathcal{I}_{1}^{j}, \mathcal{I}_{2}^{j}$, and $\mathcal{I}_{3}^{j}$, i.e. $\mathcal{I}=\mathcal{I}_{1}^{j} \cup \mathcal{I}_{2}^{j} \cup \mathcal{I}_{3}^{j}$, such that

$$
\begin{aligned}
& \mathcal{I}_{1}^{j}:=\left\{i \in \mathcal{I}: d_{\omega, i}^{j}=y_{\omega}^{j}-\left(h_{\omega, i}^{j}-f_{\omega, i}^{j} x\right)\right\}, \\
& \mathcal{I}_{2}^{j}:=\left\{i \in \mathcal{I}: d_{\omega, i}^{j}=\left(h_{\omega, i}^{j}-f_{\omega, i}^{j} x\right)-y_{\omega}^{j}\right\}, \text { and } \\
& \mathcal{I}_{3}^{j}:=\left\{i \in \mathcal{I}: d_{\omega, i}^{j}=\left(1-2 \mu_{\omega, i}^{j}\right)\left(y_{\omega}^{j}-\left\lfloor h_{\omega, i}^{j}\right\rfloor+f_{\omega, i}^{j} x\right)+\mu_{\omega, i}^{j}\right\} .
\end{aligned}
$$


Therefore, in the light of the above discussed cases, we can rewrite $\mathcal{K}_{\omega}^{4}(x)$ as

$$
\begin{aligned}
\mathcal{K}_{\omega}^{4}(x)= & \left\{\left(y_{\omega}, d_{\omega}\right) \in \mathbb{R}^{|J|} \times \mathbb{R}_{+}^{m_{2}|J|+|J|}: W_{\omega} y_{\omega} \geq r_{\omega}-T_{\omega} x,\right. \\
& y_{\omega}^{j} \geq\left[h_{\omega, i}^{j}\right]-f_{\omega, i}^{j} x, d_{\omega, i}^{j}=y_{\omega}^{j}-\left(h_{\omega, i}^{j}-f_{\omega, i}^{j} x\right), i \in \mathcal{I}_{1}^{j}, j \in J, \\
& y_{\omega}^{j} \leq\left\lfloor h_{\omega, i}^{j}\right\rfloor-f_{\omega, i}^{j} x, d_{\omega, i}^{j}=\left(h_{\omega, i}^{j}-f_{\omega, i}^{j} x\right)-y_{\omega}^{j}, i \in \mathcal{I}_{2}^{j}, j \in J, \quad i \in \mathcal{I}_{3}^{j}, j \in J, \\
& \left\lfloor h_{\omega, i}^{j}\right\rfloor-f_{\omega, i}^{j} x \leq y_{\omega}^{j} \leq\left\lceil h_{\omega, i}^{j}\right\rceil-f_{\omega, i}^{j} x, \quad \\
& \left.d_{\omega, i}^{j}=\left(1-2 \mu_{\omega, i}^{j}\right)\left(y_{\omega}^{j}-\left\lfloor h_{\omega, i}^{j}\right\rfloor+f_{\omega, i}^{j} x\right)+\mu_{\omega, i}^{j}, i \in \mathcal{I}_{3}^{j}, j \in J\right\} .
\end{aligned}
$$

The compact form $\mathcal{K}_{\omega}^{4}(x)$ can be written as

$$
\begin{aligned}
\mathcal{K}_{\omega}^{4}(x)= & \left\{\left(y_{\omega}, d_{\omega}\right) \in \mathbb{R}^{|J|} \times \mathbb{R}_{+}^{m_{2}|J|+|J|}: W_{\omega} y_{\omega} \geq r_{\omega}-T_{\omega} x,\right. \\
& \left.d_{\omega, i}^{j}=\theta_{\omega, i}^{j} y_{\omega}^{j}+\sigma_{\omega, i}^{j}, l_{\omega}^{j} \leq y_{\omega}^{j} \leq \bar{u}_{\omega}^{j}, i=1, \ldots, m_{2}, j \in J\right\},
\end{aligned}
$$

where $\theta_{\omega, i}^{j}, \sigma_{\omega, i}^{j} \in \mathbb{R}$ and $l_{\omega}^{j}, \bar{u}_{\omega}^{j} \in \mathbb{Z} \cup\{-\infty,+\infty\}$ for $j \in J$ and $i=1, \ldots, m_{2}$. Since $W_{\omega}$ is a TU matrix (by assumption), transpose of $\left(W_{\omega}^{T}, \mathbf{I},-\mathbf{I}\right)$ is also a TU matrix. Therefore, each bounded face $\mathcal{K}_{\omega}^{4}(x)$ of $\operatorname{Proj}_{y_{\omega}, d_{1}, \ldots, d_{|\Omega|} \mid} \mathcal{K}_{\omega}^{3}(x)$, for $x \in X$, has extreme points with integral $y_{\omega}^{j}$ as $r_{\omega}-T_{\omega} x$ is integral. Since $\mathcal{K}_{\omega}^{3}(x)$ is a subset of the continuous relaxation of $\mathcal{K}_{\omega}^{1}(x)$, and all bounded faces of $\mathcal{K}_{\omega}^{3}(x)$ have extreme points with integral $y_{\omega}$ components, $\mathcal{K}_{\omega}^{3}(x) \subseteq \operatorname{conv}\left(\mathcal{K}_{\omega}^{1}(x)\right)$. Hence, $\operatorname{conv}\left(\mathcal{K}_{\omega}^{1}(x)\right)=\mathcal{K}_{\omega}^{3}(x)$ for all $x \in X$ because of (45).

Finally, because of Theorem $4, \operatorname{conv}\left(\mathcal{K}_{\omega}^{1}(x)\right) \cap \mathcal{K}_{\omega}^{2}(x) \subseteq \mathcal{K}_{\omega}^{3}(x) \cap \mathcal{K}_{\omega}^{2}(x)$ where $\mathcal{K}_{\omega}^{2}(x):=$ $\left\{\left(y_{\omega}, d_{\omega}\right) \in \mathbb{R}^{|J|} \times \mathbb{R}_{+}^{m_{2}|J|+|J|}:\left\|d_{\omega}^{j}\right\|_{p} \leq d_{\omega, 0}^{j}, j \in J\right\}$, provides the convex hull (for $p=1$ ) or an approximation (for $p \geq 2$ ) for $\left(\mathcal{K}_{\omega}(x)\right)$. In other words, we obtain the convex hull (for $p=1$ ) or an approximation (for $p \geq 2$ ) of $\mathcal{K}_{\omega}(x)$ by adding $m_{2} \times|J|$ number of linear inequalities:

$$
d_{\omega, i}^{j} \geq\left(1-2 \mu_{\omega, i}^{j}\right)\left(y_{\omega}^{j}-\left\lfloor h_{\omega, i}^{j} x\right\rfloor+f_{\omega, i}^{j}\right)+\mu_{\omega, i}^{j}, \quad i=1, \ldots, m_{2}, j \in J,
$$

to the continuous relaxation of $\mathcal{K}_{\omega}(x)$. Moreover, $\mathcal{K}_{\omega}^{3}(x) \cap \mathcal{K}_{\omega}^{2}(x)$ when projected to $\left(y_{\omega}, d_{\omega, 0}\right)$ space gives the convex hull of the feasible region of $\overline{\mathcal{Q}}_{\omega}(x)$, i.e.

$$
\begin{aligned}
& \left\{\left(y_{\omega}, d_{\omega, 0}\right) \in \mathbb{R}^{|J|} \times \mathbb{R}_{+}^{|J|}:(33),(34),\right. \text { and } \\
& \left.\left\|\bar{E}_{\omega, l}^{j} y_{\omega}^{j}+\bar{F}_{\omega, l}^{j} x-\bar{h}_{\omega, l}^{j}\right\|_{p} \leq d_{\omega, 0}^{j}, \quad l \in \mathcal{L}, j \in J\right\},
\end{aligned}
$$

where the $i$ th row of $\bar{E}_{\omega, l}^{j} y_{\omega}^{j}+\bar{F}_{\omega, l}^{j} x-\bar{h}_{\omega, l}^{j}$ is either $y_{\omega}^{j}+f_{\omega, i}^{j} x-h_{\omega, i}^{j}$ or $\left(1-2 \mu_{\omega, i}^{j}\right)$ $\left(y_{\omega}^{j}-\left\lfloor h_{\omega, i}^{j}\right\rfloor+f_{\omega, i}^{j} x\right)+\mu_{\omega, i}^{j}$. This completes the proof.

Next, we consider multi-integer generalization of the second stage problem studied in Theorem 6. More specifically, we again consider second stage problems with $|J|$ conic constraints (54), but each constraint has $q$ integer variables $y_{\omega}^{j} \in \mathbb{Z}^{q}$ associated to it and these integer variables $y_{\omega}=\left(y_{\omega}^{1}, y_{\omega}^{2}, \ldots, y_{\omega}^{|J|}\right) \in \mathbb{Z}^{q|J|}$ are also connected with linear constraints (53). 
Theorem 7 In TSS-CMIP (1) and TSDR-CMIP (9), let

$$
\begin{array}{rll}
\overline{\mathcal{Q}}_{\omega}(x):=\min & g_{\omega} y_{\omega}+\sum_{j \in J} \hat{g}_{\omega}^{j} d_{\omega, 0}^{j} & \\
\text { s.t. } & W_{\omega} y_{\omega} \geq r_{\omega}-T_{\omega} x, & \\
& \left\|E_{\omega}^{j} y_{\omega}^{j}+F_{\omega}^{j} x-h_{\omega}^{j}\right\|_{p} \leq d_{\omega, 0}^{j}, & j \in J, \\
y_{\omega}^{j} \in \mathbb{Z}^{q}, d_{\omega, 0}^{j} \in \mathbb{R}_{+}, & j \in J,
\end{array}
$$

where $r_{\omega}$ is integral and either of following conditions is satisfied:

(i) $E_{\omega}^{j}$ for $j \in J$ and $W_{\omega}$ are network flow matrices;

(ii) $E_{\omega}^{j}$ for $j \in J$ are TU matrices, and $W_{\omega}=\mathbf{I}_{m_{3}}$ or $W_{\omega}=\mathbf{0}$.

For $p=1$ or $p \geq 2$, the convex hull or an approximation, respectively, of the feasible region of $\overline{\mathcal{Q}}_{\omega}(x)$ for all $x \in X$ are given by

$$
\begin{aligned}
& \left\{\left(y_{\omega}, d_{\omega, 0}\right) \in \mathbb{R}^{q|J|} \times \mathbb{R}_{+}^{|J|}:(53),(54),\right. \text { and } \\
& \left.\left\|\bar{E}_{\omega, l}^{j} y_{\omega}^{j}+\bar{F}_{\omega, l}^{j} x-\bar{h}_{\omega, l}^{j}\right\|_{p} \leq d_{\omega, 0}^{j}, \quad l \in \mathcal{L}, j \in J\right\},
\end{aligned}
$$

where the ith element of $\bar{E}_{\omega, l}^{j} y_{\omega}^{j}+\bar{F}_{\omega, l}^{j} x-\bar{h}_{\omega, l}^{j}$ is either $e_{\omega, i}^{j} y_{\omega}^{j}+f_{\omega, i}^{j} x-h_{\omega, i}^{j}$ or $\left(1-2 \mu_{\omega, i}^{j}\right)\left(e_{\omega, i}^{j} y_{\omega}^{j}-\left\lfloor h_{\omega, i}^{j}\right\rfloor+f_{\omega, i}^{j} x\right)+\mu_{\omega, i}^{j}$, and $\mu_{\omega, i}^{j}=h_{\omega, i}^{j}-\left\lfloor h_{\omega, i}^{j}\right\rfloor$. Furthermore, in higher dimensional space $\overline{\mathcal{Q}}_{\omega}(x)$ can be reformulated as:

$$
\begin{array}{rlrl}
\mathcal{Q}_{\omega}(x):= & \min \left\{g_{\omega} y_{\omega}+\sum_{j \in J} \hat{g}_{\omega}^{j} d_{\omega, 0}^{j}:\right. \text { (53), and } & \\
& \left|e_{\omega, i}^{j} y_{\omega}^{j}+f_{\omega, i}^{j} x-h_{\omega, i}^{j}\right| \leq d_{\omega, i}^{j}, & i=1, \ldots, m_{2}, j \in J, \\
\left\|d_{\omega}^{j}\right\|_{p} \leq d_{\omega, 0}^{j}, & j \in J, \\
y_{\omega}^{j} \in \mathbb{Z}^{q}, d_{\omega, 0}^{j} \in \mathbb{R}_{+}, d_{\omega}^{j} \in \mathbb{R}_{+}^{m_{2}}, & j \in J\} .
\end{array}
$$

Then, for all $x \in X$, the convex hull (for $p=1$ ) and an approximation (for $p \geq 2$ ) of the feasible region of $\mathcal{Q}_{\omega}(x)$, denoted by $\mathcal{K}_{\omega}(x)$, is obtained by adding $m_{2} \times|J|$ number of the following linear inequalities (in the higher dimensional space) to the continuous relaxation of $\mathcal{K}_{\omega}(x)$ :

$$
\left(1-2 \mu_{\omega, i}^{j}\right)\left(e_{\omega, i}^{j} y_{\omega}^{j}-\left\lfloor h_{\omega, i}^{j}\right\rfloor+f_{\omega, i}^{j} x\right)+\mu_{\omega, i}^{j} \leq d_{\omega, i}^{j}, \quad i=1, \ldots, m_{2}, \quad j \in J .
$$

\section{Proof Let}

$$
\begin{aligned}
\mathcal{K}_{\omega}^{1}(x)= & \left\{\left(y_{\omega}, d_{\omega}\right) \in \mathbb{R}^{q|J|} \times \mathbb{R}_{+}^{m_{2}|J|+|J|}:\right. \\
& W_{\omega} y_{\omega} \geq r_{\omega}-T_{\omega} x \\
& \left.\left|e_{\omega, i}^{j} y_{\omega}^{j}+f_{\omega, i}^{j} x-h_{\omega, i}^{j}\right| \leq d_{\omega, i}^{j}, \quad i=1, \ldots, m_{2}, \quad j \in J\right\}
\end{aligned}
$$


for all $x \in X$. Since $E_{\omega}^{j}$ is either a network flow matrix, or a TU matrix, $e_{\omega, i}^{j} y_{\omega}^{j}$ is integral. Similar to the proof of the previous theorem, we again apply Proposition 1 to each defining inequality (61) of $\mathcal{K}_{\omega}^{1}(x)$. In this case we substitute $\sigma=e_{\omega, i}^{j} y_{\omega}^{j} \in \mathbb{Z}, \beta=h_{\omega, i}^{j}-f_{\omega, i}^{j} x$, and $\rho_{1}=d_{\omega, i}^{j}$ in $Z_{1}$ and get the following valid parametric conic MIR inequalities (20) for $\mathcal{K}_{\omega}^{1}(x):\left(1-2 \mu_{\omega, i}^{j}\right)\left(e_{\omega, i}^{j} y_{\omega}^{j}-\left\lfloor h_{\omega, i}^{j}-f_{\omega, i}^{j} x\right\rfloor\right)+\mu_{\omega, i}^{j} \leq d_{\omega, i}^{j}$, for $i=1, \ldots, m_{2}$ and $j \in J$, which is equivalent to

$$
\left(1-2 \mu_{\omega, i}^{j}\right)\left(e_{\omega, i}^{j} y_{\omega}^{j}-\left\lfloor h_{\omega, i}^{j}\right\rfloor+f_{\omega, i}^{j} x\right)+\mu_{\omega, i}^{j} \leq d_{\omega, i}^{j},
$$

for $i=1, \ldots, m_{2}$ and $j \in J$, as $f_{\omega, i}^{j} x$ is integral for all $x \in X$. Thus, conv $\left(\mathcal{K}_{\omega}^{1}(x)\right) \subseteq \mathcal{K}_{\omega}^{3}(x)$ for all $x \in X$, where

$$
\begin{aligned}
\mathcal{K}_{\omega}^{3}(x)= & \left\{\left(y_{\omega}, d_{\omega}\right) \in \mathbb{R}^{q|J|} \times \mathbb{R}_{+}^{m_{2}|J|+|J|}: W_{\omega} y_{\omega} \geq r_{\omega}-T_{\omega} x,\right. \\
& d_{\omega, i}^{j} \geq e_{\omega, i}^{j} y_{\omega}^{j}-\left(h_{\omega, i}^{j}-f_{\omega, i}^{j} x\right), \quad i=1, \ldots, m_{2}, j \in J, \\
& d_{\omega, i}^{j} \geq\left(h_{\omega, i}^{j}-f_{\omega, i}^{j} x\right)-e_{\omega, i}^{j} y_{\omega}^{j}, \quad i=1, \ldots, m_{2}, j \in J, \\
d_{\omega, i}^{j} \geq & \left.\left(1-2 \mu_{\omega, i}^{j}\right)\left(e_{\omega, i}^{j} y_{\omega}^{j}-\left\lfloor h_{\omega, i}^{j}\right\rfloor+f_{\omega, i}^{j} x\right)+\mu_{\omega, i}^{j}, i=1, \ldots, m_{2}, j \in J\right\} .
\end{aligned}
$$

Notice that $\mathcal{K}_{\omega}^{3}(x)$ is a subset of the continuous relaxation of $\mathcal{K}_{\omega}^{1}(x)$, and there is no restriction on variables $d_{\omega, 0}$ for all $\omega \in \Omega$. Therefore, if we can prove that all bounded faces of $\operatorname{Proj}_{y_{\omega}, d_{1}, \ldots, d_{|\Omega|} \mid}\left(\mathcal{K}_{\omega}^{3}(x)\right)$ have extreme points with integral $y_{\omega}$ component, then it will imply that $\mathcal{K}_{\omega}^{3}(x) \subseteq \operatorname{conv}\left(\mathcal{K}_{\omega}^{1}(x)\right)$ for all $x \in X$. So, we now consider a bounded face of $\mathcal{K}_{\omega}^{3}(x)$ with maximum possible dimension, denoted by $\mathcal{K}_{\omega}^{4}(x)$, and on this face, $d_{\omega, i}^{j}$, $i=1, \ldots, m_{2}$ and $j \in J$, is minimal, i.e. $d_{\omega, i}^{j}=\max \left\{z_{\omega, i}^{j}-h_{\omega, i}^{j}, h_{\omega, i}^{j}-z_{\omega, i}^{j},(1-\right.$ $\left.\left.2 \mu_{\omega, i}^{j}\right)\left(z_{\omega, i}^{j}-\left\lfloor h_{\omega, i}^{j}\right\rfloor\right)+\mu_{\omega, i}^{j}\right\}$ where $z_{\omega, i}^{j}=e_{\omega, i}^{j} y_{\omega}^{j}+f_{\omega, i}^{j} x \in \mathbb{Z}$. Now if $h_{\omega, i}^{j} \in \mathbb{Z}$ for any $j \in J$, then $\mu_{\omega, i}^{j}=0$ and as a result inequality (62) reduces to inequality (63). However, when $h_{\omega, i}^{j} \notin \mathbb{Z}$ for some $j \in J$, then again there are three possible cases:

Case I. $d_{\omega, i}^{j}=z_{\omega, i}^{j}-h_{\omega, i}^{j}$ : This case will happen if and only if $z_{\omega, i}^{j}-h_{\omega, i}^{j} \geq h_{\omega, i}^{j}-z_{\omega, i}^{j}$ and $z_{\omega, i}^{j}-h_{\omega, i}^{j} \geq\left(1-2 \mu_{\omega, i}^{j}\right)\left(z_{\omega, i}^{j}-\left\lfloor h_{\omega, i}^{j}\right\rfloor\right)+\mu_{\omega, i}^{j}$, which are equivalent to $z_{\omega, i}^{j} \geq h_{\omega, i}^{j}$ and $z_{\omega, i}^{j} \geq\left\lceil h_{\omega, i}^{j}\right\rceil$, respectively. Therefore, we can claim that $d_{\omega, i}^{j}=z_{\omega, i}^{j}-h_{\omega, i}^{j}$ if and only if $z_{\omega, i}^{j} \geq\left\lceil h_{\omega, i}^{j}\right\rceil$.

Case II. $d_{\omega, i}^{j}=h_{\omega, i}^{j}-z_{\omega, i}^{j}$ : This case will happen if and only if $h_{\omega, i}^{j}-z_{\omega, i}^{j} \geq z_{\omega, i}^{j}-h_{\omega, i}^{j}$ and $h_{\omega, i}^{j}-z_{\omega, i}^{j} \geq\left(1-2 \mu_{\omega, i}^{j}\right)\left(z_{\omega, i}^{j}-\left\lfloor h_{\omega, i}^{j}\right\rfloor\right)+\mu_{\omega, i}^{j}$, which are equivalent to $z_{\omega, i}^{j} \leq h_{\omega, i}^{j}$ and $z_{\omega, i}^{j} \leq\left\lfloor h_{\omega, i}^{j}\right\rfloor$, respectively, as $\mu_{\omega, i}^{j} \leq 1$. Therefore, $d_{\omega, i}^{j}=h_{\omega, i}^{j}-z_{\omega, i}^{j}$ if and only if $z_{\omega, i}^{j} \leq\left\lfloor h_{\omega, i}^{j}\right\rfloor$.

Case III. $d_{\omega, i}^{j}=\left(1-2 \mu_{\omega, i}^{j}\right)\left(z_{\omega, i}^{j}-\left\lfloor h_{\omega, i}^{j}\right\rfloor\right)+\mu_{\omega, i}^{j}$ : This case will happen if and only if $\left(1-2 \mu_{\omega, i}^{j}\right)\left(z_{\omega, i}^{j}-\left\lfloor h_{\omega, i}^{j}\right\rfloor\right)+\mu_{\omega, i}^{j} \geq h_{\omega, i}^{j}-z_{\omega, i}^{j}$ and $\left(1-2 \mu_{\omega, i}^{j}\right)\left(z_{\omega, i}^{j}-\left\lfloor h_{\omega, i}^{j}\right\rfloor\right)+$ 
$\mu_{\omega, i}^{j} \geq z_{\omega, i}^{j}-h_{\omega, i}^{j}$, which are equivalent to $z_{\omega, i}^{j} \geq\left\lfloor h_{\omega, i}^{j}\right\rfloor$ and $z_{\omega, i}^{j} \leq\left\lceil h_{\omega, i}^{j}\right\rceil$, respectively. Therefore, $d_{\omega, i}^{j}=\left(1-2 \mu_{\omega, i}^{j}\right)\left(z_{\omega, i}^{j}-\left\lfloor h_{\omega, i}^{j}\right\rfloor\right)+\mu_{\omega, i}^{j}$ if and only if $\left\lfloor h_{\omega, i}^{j}\right\rfloor \leq z_{\omega, i}^{j} \leq$ $\left\lceil h_{\omega, i}^{j}\right\rceil$.

For each $j \in J$, let $\mathcal{I}:=\left\{1, \ldots, m_{2}\right\}$ be partitioned into the disjoint sets $\mathcal{I}_{1}^{j}, \mathcal{I}_{2}^{j}$, and $\mathcal{I}_{3}^{j}$, i.e. $\mathcal{I}=\mathcal{I}_{1}^{j} \cup \mathcal{I}_{2}^{j} \cup \mathcal{I}_{3}^{j}$, such that $\mathcal{I}_{1}^{j}:=\left\{i \in \mathcal{I}: d_{\omega, i}^{j}=z_{\omega, i}^{j}-h_{\omega, i}^{j}\right\}, \mathcal{I}_{2}^{j}:=\left\{i \in \mathcal{I}: d_{\omega, i}^{j}=\right.$ $\left.h_{\omega, i}^{j}-z_{\omega, i}^{j}\right\}$, and $\mathcal{I}_{3}^{j}:=\left\{i \in \mathcal{I}: d_{\omega, i}^{j}=\left(1-2 \mu_{\omega, i}^{j}\right)\left(z_{\omega, i}^{j}-\left\lfloor h_{\omega, i}^{j}\right\rfloor\right)+\mu_{\omega, i}^{j}\right\}$. Therefore, in the light of the above discussed cases, we can define $\mathcal{K}_{\omega}^{4}(x)$ as

$$
\begin{aligned}
& \mathcal{K}_{\omega}^{4}(x)=\left\{\left(y_{\omega}, d_{\omega}\right) \in \mathbb{R}^{q|J|} \times \mathbb{R}_{+}^{m_{2}|J|+|J|}: W_{\omega} y_{\omega} \geq r_{\omega}-T_{\omega} x,\right. \\
& e_{\omega, i}^{j} y_{\omega}^{j} \geq\left[h_{\omega, i}^{j}\right]-f_{\omega, i}^{j} x, d_{\omega, i}^{j}=e_{\omega, i}^{j} y_{\omega}^{j}-\left(h_{\omega, i}^{j}-f_{\omega, i}^{j} x\right), i \in \mathcal{I}_{1}^{j}, j \in J, \\
& e_{\omega, i}^{j} y_{\omega}^{j} \leq\left\lfloor h_{\omega, i}^{j}\right\rfloor-f_{\omega, i}^{j} x, d_{\omega, i}^{j}=\left(h_{\omega, i}^{j}-f_{\omega, i}^{j} x\right)-e_{\omega, i}^{j} y_{\omega}^{j}, i \in \mathcal{I}_{2}^{j}, j \in J, \\
& \left\lfloor h_{\omega, i}^{j}\right\rfloor-f_{\omega, i}^{j} x \leq e_{\omega, i}^{j} y_{\omega}^{j} \leq\left[h_{\omega, i}^{j}\right]-f_{\omega, i}^{j} x, \quad i \in \mathcal{I}_{3}^{j}, j \in J, \\
& \left.d_{\omega, i}^{j}=\left(1-2 \mu_{\omega, i}^{j}\right)\left(e_{\omega, i}^{j} y_{\omega}^{j}-\left\lfloor h_{\omega, i}^{j}\right\rfloor+f_{\omega, i}^{j} x\right)+\mu_{\omega, i}^{j}, \quad i \in \mathcal{I}_{3}^{j}, j \in J\right\} .
\end{aligned}
$$

Let $\left(\hat{y}_{\omega}, \hat{d}_{\omega}\right)$ be an extreme point of $\operatorname{Proj}_{y_{\omega}, d_{1}, \ldots, d_{|\Omega|} \mid}\left(\mathcal{K}_{\omega}^{4}(x)\right)$ for a given $x \in X$, which is on the intersection of $|J|\left(q+m_{2}\right)$ constraints of $\mathcal{K}_{\omega}^{4}(x)$ such that the matrix defining these constraints is nonsingular. Since the polyhedron $\mathcal{K}_{\omega}^{4}(x)$ already has $m_{2}|J|$ equality constraints, we need a combination of at least $q|J|$ defining inequalities of $\mathcal{K}_{\omega}^{4}(x)$ to be binding at the point $\left(\hat{y}_{\omega}, \hat{d}_{\omega}\right)$. Let the constraints $w_{\omega, i} y_{\omega} \geq r_{\omega, i}-t_{\omega, i} x$ for $i \in \tau_{0}, e_{\omega, i}^{j} y_{\omega}^{j} \geq\left\lceil h_{\omega, i}^{j}\right\rceil-f_{\omega, i}^{j} x$, for $i \in \tau_{1}^{j}, j \in J_{1}$, and $e_{\omega, i}^{j} y_{\omega}^{j} \leq\left\lfloor h_{\omega, i}^{j}\right\rfloor-f_{\omega, i}^{j} x$ for $i \in \tau_{2}^{j}, j \in J_{2}$ be binding at the point $\left(\hat{y}_{\omega}, \hat{d}_{\omega}\right)$ where $w_{\omega, i}, r_{\omega, i}$, and $t_{\omega, i}$ denote $i$ th row of matrix/vector $W_{\omega}, r_{\omega}$, and $T_{\omega}$, respectively, and $\tau_{0}, \tau_{1}^{j}, j \in J_{1} \subseteq J$, and $\tau_{2}^{j}, j \in J_{2} \subseteq J$, are subsets of $\left\{1, \ldots, m_{2}\right\}$ such that $\left|\tau_{0}\right|+\sum_{j \in J_{1}}\left|\tau_{1}^{j}\right|+\sum_{j \in J_{2}}\left|\tau_{2}^{j}\right|=q|J|$. Then, $\hat{y}_{\omega}$ satisfies the following system of equations:

$$
\begin{array}{ll}
w_{\omega, i} \hat{y}_{\omega}=r_{\omega, i}-t_{\omega, i} x, & i \in \tau_{0}, \\
e_{\omega, i}^{j} \hat{y}_{\omega}^{j}=\left\lceil h_{\omega, i}^{j}\right\rceil-f_{\omega, i}^{j} x, & i \in \tau_{1}^{j}, j \in J_{1}, \\
e_{\omega, i}^{j} \hat{y}_{\omega}^{j}=\left\lfloor h_{\omega, i}^{j}\right\rfloor-f_{\omega, i}^{j} x, & i \in \tau_{2}^{j}, j \in J_{2},
\end{array}
$$

which in compact form is written as $\Pi_{\omega} \hat{y}_{\omega}=\pi_{\omega}(x)$, where $\Pi_{\omega}$ is a submatrix of

$$
\left[\begin{array}{c}
W_{\omega} \\
E_{\omega}
\end{array}\right]=\left[\begin{array}{ccc}
W_{\omega}^{1} & \cdots & W_{\omega}^{|J|} \\
E_{\omega}^{1} & 0 & 0 \\
0 & \ddots & 0 \\
0 & 0 & E_{\omega}^{|J|}
\end{array}\right] .
$$

According to condition (i), if $W_{\omega}$ and $E_{\omega}^{j}$ are network flow matrices, i.e., each row of these matrices represents arc in a network and each column corresponds to node, then $\left[\begin{array}{c}W_{\omega} \\ E_{\omega}\end{array}\right]$ 
is equivalent to adding arcs in a digraph, which is still a network flow matrix and is TU ( [42]). Thus, $\Pi_{\omega}$ is also TU. Similarly, for condition (ii) when $E_{\omega}^{j}$ for $j \in J$ are TU matrices, which implies $E_{\omega}$ is TU, and $W_{\omega}=\mathbf{I}_{m_{3}}$ (or $W_{\omega}=\mathbf{0}$ ), $\Pi_{\omega}$ is a TU matrix.

Also, since each component of the vector $\pi_{\omega}(x)$, i.e., $r_{\omega, i}-t_{\omega, i} x,\left\lceil h_{\omega, i}^{j}\right\rceil-f_{\omega, i}^{j} x$, or $\left\lfloor h_{\omega, i}^{j}\right\rfloor-f_{\omega, i}^{j} x$, is integral, $\hat{y}_{\omega}$ is integral. Hence, all bounded faces of $\mathcal{K}_{\omega}^{3}(x)$ have extreme point $\left(\hat{y}_{\omega}, \hat{d}_{\omega}\right)$ in $\operatorname{Proj}_{y_{\omega}, d_{1}, \ldots, d_{|\Omega|}}\left(\mathcal{K}_{\omega}^{3}(x)\right)$ space with integral $\hat{y}_{\omega}$ and since $\mathcal{K}_{\omega}^{3}(x)$ is a subset of the continuous relaxation of $\mathcal{K}_{\omega}^{1}(x), \mathcal{K}_{\omega}^{3}(x) \subseteq \operatorname{conv}\left(\mathcal{K}_{\omega}^{1}(x)\right)$ for all $x \in X$. Therefore, we have $\mathcal{K}_{\omega}^{3}(x)=\operatorname{conv}\left(\mathcal{K}_{\omega}^{1}(x)\right)$.

Finally because of Theorem $4, \operatorname{con} v\left(\mathcal{K}_{\omega}^{1}(x)\right) \cap \mathcal{K}_{\omega}^{2}(x) \subseteq \mathcal{K}_{\omega}^{3}(x) \cap \mathcal{K}_{\omega}^{2}(x)$ where $\mathcal{K}_{\omega}^{2}(x):=$ $\left\{\left(y_{\omega}, d_{\omega}\right) \in \mathbb{R}^{q|J|} \times \mathbb{R}_{+}^{m_{2}|J|+|J|}:\left\|d_{\omega}^{j}\right\|_{p} \leq d_{\omega, 0}^{j}, j \in J\right\}$, provides the convex hull (for $p=1$ ) or an approximation (for $p \geq 2$ ) for $\mathcal{K}_{\omega}(x)$. In other words, we obtain the convex hull (for $p=1$ ) or an approximation (for $p \geq 2$ ) of $\mathcal{K}_{\omega}(x)$ by adding $m_{2} \times|J|$ number of linear inequalities:

$$
d_{\omega, i}^{j} \geq\left(1-2 \mu_{\omega, i}^{j}\right)\left(e_{\omega, i}^{j} y_{\omega}^{j}-\left\lfloor h_{\omega, i}^{j}\right\rfloor+f_{\omega, i}^{j}\right)+\mu_{\omega, i}^{j}, i=1, \ldots, m_{2}, j \in J,
$$

to the continuous relaxation of $\mathcal{K}_{\omega}(x)$. Moreover, $\mathcal{K}_{\omega}^{3}(x) \cap \mathcal{K}_{\omega}^{2}(x)$ when projected to $\left(y_{\omega}, d_{\omega, 0}\right)$ space gives the convex hull (for $p=1$ ) or an approximation (for $p \geq 2$ ) of the feasible region of $\overline{\mathcal{Q}}_{\omega}(x)$, i.e.

$$
\begin{aligned}
& \left\{\left(y_{\omega}, d_{\omega, 0}\right) \in \mathbb{R}^{q|J|} \times \mathbb{R}_{+}^{|J|}:(33),(34),\right. \text { and } \\
& \left.\left\|\bar{E}_{\omega, l}^{j} y_{\omega}^{j}+\bar{F}_{\omega, l}^{j} x-\bar{h}_{\omega, l}^{j}\right\|_{p} \leq d_{\omega, 0}^{j}, \quad l \in \mathcal{L}, j \in J\right\},
\end{aligned}
$$

where the $i$ th row of $\bar{E}_{\omega, l}^{j} y_{\omega}^{j}+\bar{F}_{\omega, l}^{j} x-\bar{h}_{\omega, l}^{j}$ is either $e_{\omega, i}^{j} y_{\omega}^{j}+f_{\omega, i}^{j} x-h_{\omega, i}^{j}$ or $\left(1-2 \mu_{\omega, i}^{j}\right)\left(e_{\omega, i}^{j} y_{\omega}^{j}-\left\lfloor h_{\omega, i}^{j}\right\rfloor+f_{\omega, i}^{j} x\right)+\mu_{\omega, i}^{j}$. This completes the proof.

\subsection{Scenario-based cuts for extensive formulation of structured TSS-CMIPs}

We consider extensive formulation of structured TSS-CMIPs introduced in the previous section and present partial convex hull for them using Theorems 5-7 and Corollary 1; the proofs are provided in "Appendix C". Note that for $\hat{x} \in X$ and $\omega \in \Omega, \overline{\mathcal{K}}_{\omega}(\hat{x})=\operatorname{Proj}_{x=\hat{x}, y_{\omega}, d_{\omega}}(\overline{\mathcal{P}})$ and $\overline{\mathcal{K}}_{\text {tight }}^{\omega}(\hat{x})=\operatorname{Proj}_{x=\hat{x}, y_{\omega}, d_{\omega}}\left(\overline{\mathcal{P}}_{p c h}\right)$.

\section{Corollary 2 Let}

$$
\begin{gathered}
\overline{\mathcal{P}}:=\left\{\sqrt{\left(y_{\omega, 1}+f_{\omega} x-h_{\omega}\right)^{2}+\left(y_{\omega, 2}\right)^{2}} \leq d_{\omega, 0}, \omega \in \Omega\right. \\
\left.x \in X, y_{\omega, 1} \in \mathbb{Z}, y_{\omega, 2} \in \mathbb{R}_{+}, d_{\omega, 0} \in \mathbb{R}_{+}, \quad \omega \in \Omega\right\} .
\end{gathered}
$$

For each nonempty set $\Gamma \subseteq \Omega$, a partial convex hull of $\overline{\mathcal{P}}$ is given by

$$
\overline{\mathcal{P}}_{p c h}:=\left\{\left(x, y_{\omega, 1}, y_{\omega, 2}, d_{\omega, 0}\right) \in X \times \mathbb{R} \times \mathbb{R}_{+}^{2}:\right. \text { (69) and }
$$




$$
\left.\sqrt{\left(\left(1-2 \mu_{\omega}\right)\left(y_{\omega, 1}+f_{\omega} x-h_{\omega}\right)+\mu_{\omega}\right)^{2}+\left(y_{\omega, 2}\right)^{2}} \leq d_{\omega, 0}, \quad \omega \in \Omega\right\},
$$

where $\mu_{\omega}=h_{\omega}-\left\lfloor h_{\omega}\right\rfloor$.

\section{Corollary 3 Let}

$$
\begin{gathered}
\overline{\mathcal{P}}:=\left\{\left(x,\left\{y_{\omega}, d_{\omega, 0}\right\}_{\omega \in \Omega}\right) \in X \times \mathbb{Z}^{|\Omega| \times|J|} \times \mathbb{R}_{+}^{|\Omega| \times|J|}: T_{\omega} x+W_{\omega} y_{\omega} \geq r_{\omega}, \omega \in \Omega,\right. \\
\left.\left\|\mathbf{1} y_{\omega}^{j}+F_{\omega}^{j} x-h_{\omega}^{j}\right\|_{p} \leq d_{\omega, 0}^{j}, j \in J, \omega \in \Omega\right\},
\end{gathered}
$$

where $W_{\omega}$ is a TU matrix. For each nonempty set $\Gamma \subseteq \Omega$, a partial convex hull of $\overline{\mathcal{P}}$ with $p=1$ is given by

$$
\begin{aligned}
\overline{\mathcal{P}}_{p c h}: & =\left\{\left(x,\left\{y_{\omega}, d_{\omega, 0}\right\}_{\omega \in \Omega}\right) \in X \times\left(\mathbb{Z}^{|\Omega|-|\Gamma|} \times \mathbb{R}^{|\Gamma|}\right)^{|J|} \times \mathbb{R}_{+}^{|\Omega| \times|J|}:\right. \\
& T_{\omega} x+W_{\omega} y_{\omega} \geq r_{\omega}, \omega \in \Omega \\
& \left\|\mathbf{1} y_{\omega}^{j}+F_{\omega}^{j} x-h_{\omega}^{j}\right\|_{p} \leq d_{\omega, 0}^{j}, j \in J, \omega \in \Omega, \\
& \left.\left\|\bar{E}_{\omega, l}^{j} y_{\omega}^{j}+\bar{F}_{\omega, l}^{j} x-\bar{h}_{\omega, l}^{j}\right\|_{p} \leq d_{\omega, 0}^{j}, l \in \mathcal{L}, j \in J, \omega \in \Gamma\right\},
\end{aligned}
$$

where the ith row of $\bar{E}_{\omega, l}^{j} y_{\omega}^{j}+\bar{F}_{\omega, l}^{j} x-\bar{h}_{\omega, l}^{j}$ is either $y_{\omega}^{j}+f_{\omega, i}^{j} x-h_{\omega, i}^{j}$ or $\left(1-2 \mu_{\omega, i}^{j}\right)$ $\left(y_{\omega}^{j}-\left\lfloor h_{\omega, i}^{j}\right\rfloor+f_{\omega, i}^{j} x\right)+\mu_{\omega, i}^{j}$, and $\mu_{\omega, i}^{j}=h_{\omega, i}^{j}-\left\lfloor h_{\omega, i}^{j}\right\rfloor$.

\section{Corollary 4 Let}

$$
\begin{aligned}
\overline{\mathcal{P}}:= & \left\{\left(x,\left\{y_{\omega}, d_{\omega, 0}\right\}_{\omega \in \Omega}\right) \in X \times \mathbb{Z}^{q|\Omega| \times|J|} \times \mathbb{R}_{+}^{|\Omega| \times|J|}:\right. \\
& T_{\omega} x+W_{\omega} y_{\omega} \geq r_{\omega}, \omega \in \Omega, \\
& \left.\left\|E_{\omega}^{j} y_{\omega}^{j}+F_{\omega}^{j} x-h_{\omega}^{j}\right\|_{p} \leq d_{\omega, 0}^{j}, j \in J, \omega \in \Omega\right\},
\end{aligned}
$$

such that either of following conditions is satisfied:

(i) $E_{\omega}^{j}, j \in J$, and $W_{\omega}$ are network flow matrices;

(ii) $E_{\omega}^{j}, j \in J$ are TU matrices, and $W_{\omega}=\mathbf{I}_{m_{3}}$ or $W_{\omega}=\mathbf{0}$.

For each nonempty set $\Gamma \subseteq \Omega$, a partial convex hull of $\overline{\mathcal{P}}$ with $p=1$ is given by

$$
\begin{aligned}
\overline{\mathcal{P}}_{p c h}: & =\left\{\left(x,\left\{y_{\omega}, d_{\omega, 0}\right\}_{\omega \in \Omega}\right) \in X \times\left(\mathbb{Z}^{|\Omega|-|\Gamma|} \times \mathbb{R}^{|\Gamma|}\right)^{q \times|J|} \times \mathbb{R}_{+}^{|\Omega| \times|J|}:\right. \\
& T_{\omega} x+W_{\omega} y_{\omega} \geq r_{\omega}, \omega \in \Omega, \\
& \left\|E_{\omega}^{j} y_{\omega}^{j}+F_{\omega}^{j} x-h_{\omega}^{j}\right\|_{p} \leq d_{\omega, 0}^{j}, j \in J, \omega \in \Omega, \\
& \left.\left\|\bar{E}_{\omega, l}^{j} y_{\omega}^{j}+\bar{F}_{\omega, l}^{j} x-\bar{h}_{\omega, l}^{j}\right\|_{p} \leq d_{\omega, 0}^{j}, l \in \mathcal{L}, j \in J, \omega \in \Gamma\right\},
\end{aligned}
$$

where the ith row of $\bar{E}_{\omega, l}^{j} y_{\omega}^{j}+\bar{F}_{\omega, l}^{j} x-\bar{h}_{\omega, l}^{j}$ is either $e_{\omega, i}^{j} y_{\omega}^{j}+f_{\omega, i}^{j} x-h_{\omega, i}^{j}$ or $\left(1-2 \mu_{\omega, i}^{j}\right)\left(e_{\omega, i}^{j} y_{\omega}^{j}-\left\lfloor h_{\omega, i}^{j}\right\rfloor+f_{\omega, i}^{j} x\right)+\mu_{\omega, i}^{j}$, and $\mu_{\omega, i}^{j}=h_{\omega, i}^{j}-\left\lfloor h_{\omega, i}^{j}\right\rfloor$. 


\subsection{Tight second stage formulation and partial convex hull for the extensive formulation of MM-SFLP-S}

We also provide tight second stage formulation for MM-SFLP-S (introduced in Sect. 1.3) and partial convex hull for the extensive formulation of the MM-SFLP-S. To do so, we first reformulate the second stage problem (16)-(18) using additional continuous variables (as discussed in Sect. 4) to get

$$
\begin{aligned}
Q_{\omega}(x, z, u)=\min & \sum_{i \in P} w_{i n v}^{i}\left(\sum_{k=1}^{n} \alpha_{k} x_{i k}+u_{i}-\sum_{j \in P^{\prime}}\left(z_{i j}+y_{\omega}^{i j}\right)\right) \\
& +w_{s c} \sum_{j \in P^{\prime}} \sum_{i \in P} t_{i j} y_{i j}^{\omega}+w_{p e n} d_{\omega, 0} \\
\text { s.t. } & \left|\sum_{i \in P}\left(y_{\omega}^{i j}+z_{i j}\right)-\zeta_{\omega}^{j}\right| \leq d_{\omega, j}, \text { for } j \in P^{\prime}, \\
& \sum_{j \in P^{\prime}}\left|d_{\omega, j}\right| \leq d_{\omega, 0}, \quad d_{\omega, j} \geq 0, j \in P^{\prime}, \quad \text { (17), (18) hold. }
\end{aligned}
$$

Corollary 5 Let $\mathcal{Y}_{\omega}(x, z, u):=\left\{y_{\omega}:(71)-(72)\right\}$. For each $(x, z, u) \in \mathcal{X}$ and $\omega \in \Omega$, the convex hull of $\mathcal{Y}_{\omega}(x, z, u)$ is obtained by adding the following scenario-based conic MIR cuts to the continuous relaxation of $\mathcal{Y}_{\omega}(x, z, u)$ :

$$
\left(1-2 \zeta_{\omega}^{j}+2\left\lfloor\zeta_{\omega}^{j}\right\rfloor\right)\left(\sum_{i \in P} y_{i j}^{\omega}-\left\lfloor\zeta_{\omega}^{j}\right\rfloor+\sum_{i \in P} z_{i j}\right)+\zeta_{\omega}^{j}-\left\lfloor\zeta_{\omega}^{j}\right\rfloor \leq d_{\omega, j}, \text { for all } j \in P^{\prime}
$$

Moreover, the addition of the foregoing cuts in $\left(x, z, u, y_{\omega}, d_{\omega}\right)$ space to the extensive formulation of the MM-SFLP-S provides a partial convex hull (with no integrality restrictions on $y_{\omega}$ integer variables) for the feasible region of the extensive formulation.

Proof Observe that the coefficient matrix associated with $y_{\omega}^{j}:=\left(y_{\omega}^{1 j}, y_{\omega}^{2 j}, \ldots, y_{\omega}^{|P| j}\right)$ variables in constraint (71) is a network flow matrix. Likewise, the coefficient matrix associated with $y_{\omega}$ variables in constraint (17) is a network flow matrix. Therefore, we utilize Theorem 7(i) and Corollary 4(i) to derive convex hull of $\mathcal{Y}_{\omega}(x, z, u)$ for each $(x, z, u) \in \mathcal{X}$ and partial convex hull (with no integrality restrictions on $y_{\omega}$ variables) for the extensive formulation of the MM-SFLP-S, respectively, by adding the scenario-based conic MIR cuts,

$$
\left(1-2 \zeta_{\omega}^{j}+2\left\lfloor\zeta_{\omega}^{j}\right\rfloor\right)\left(\sum_{i \in P} y_{i j}^{\omega}-\left\lfloor\zeta_{\omega}^{j}\right\rfloor+\sum_{i \in P} z_{i j}\right)+\zeta_{\omega}^{j}-\left\lfloor\zeta_{\omega}^{j}\right\rfloor \leq d_{\omega, j}, \text { for all } j \in P^{\prime}
$$

to the continuous relaxation of $\mathcal{Y}_{\omega}$ and the extensive formulation, respectively. 
Remark 2 The scenario-based cuts $(73)$ in $\left(x, z, u, y_{\omega}, d_{\omega}\right)$ space are valid for the extensive formulation of MM-DRFLP-S, i.e.,

$$
\begin{array}{ll}
\min & \sum_{i \in P} \sum_{k=1}^{n} c_{i}^{k} x_{i k}+\sum_{i \in P} \sum_{j \in P^{\prime}} t_{i j} z_{i j}+\sum_{i \in P} g_{i} u_{i}+\theta \\
\text { s.t. } & \sum_{\omega \in \Omega} \bar{p}_{\omega} f\left(x, z, u, y_{\omega}, d_{\omega}\right) \leq \theta, \quad \text { for all }\{\bar{p}\}_{\omega \in \Omega} \in \mathfrak{P}, \\
& (x, z, u) \in \mathcal{X}, \quad(71)-(72) \text { for all } \omega \in \Omega,
\end{array}
$$

where the affine function

$$
\begin{aligned}
f\left(x, z, u, y_{\omega}, d_{\omega}\right):= & \sum_{i \in P} w_{i n v}^{i}\left(\sum_{k=1}^{n} \alpha_{k} x_{i k}+u_{i}-\sum_{j \in P^{\prime}}\left(z_{i j}+y_{\omega}^{i j}\right)\right) \\
& +w_{s c} \sum_{j \in P^{\prime}} \sum_{i \in P} t_{i j} y_{i j}^{\omega}+w_{p e n} d_{\omega, 0} .
\end{aligned}
$$

\section{Computational experiments}

We perform computational experiments to evaluate the effectiveness of adding scenariobased cuts (a priori) for solving MM-SFLP-S and MM-DRFLP-S (introduced in Sect. 1.3), and structured TSS-CMIP test and TSDR-CMIP instances. We describe generation of the test instances in Sects. 7.1 and 7.2, and present our computational results in Sect. 7.3. For MM-SFLP-S and structured TSS-CMIP instances, we assume that all scenarios have same probability, i.e., $\bar{p}_{\omega}=1 /|\Omega|$ for all $\omega \in \Omega$. In contrast, for MM-DRFLP-S and structured TSDR-CMIP instances, we consider a finite set of distributions $\mathfrak{P}$ with $|\mathfrak{P}| \in\{5,10,25,50\}$. We generate the probability distributions for each ambiguity set as follows. For a probability distribution with $|\Omega|$ scenarios, we randomly draw $|\Omega|$ numbers, i.e., $R N_{\omega}$ for $\omega \in \Omega$, between $[0,10000]$, and the probability of scenario $\omega$ is calculated as $\bar{p}_{\omega}=\frac{R N_{\omega}}{\sum_{\omega \in \Omega} R N_{\omega}}$.

\subsection{Generation of MM-SFLP-S and MM-DRFLP-S test instances}

We utilize "capacitated warehouse location problem" instances from J.E. Beasley OR-Library [10] where a set $P:=\{1, \ldots, 16\}$ of facilities, a set $P^{\prime}:=\{1, \ldots, 50\}$ of retailers, and transportation cost $t_{i j}$ for each $i \in P$ and $j \in P^{\prime}$ are given. However, unlike instance in this library where demand $\mu \in \mathbb{R}_{+}^{\left|P^{\prime}\right|}$ is assumed to be deterministic, we consider uncertain demand which follows a normal distribution with $\mu$ (provided in the library) as mean and variance is randomly drawn from a uniform distribution, i.e., uniform $[0.1 \mu, 0.3 \mu]$. Moreover, instead of assuming that each facility has machines of fixed capacities, we consider $n$ different types of machines with different capacities ( $n \in\{2,4\}$ for our experiments). Unlike the warehouse location problem, the MM-SFLP-S and MM-DRFLP-S also allow subcontracting and last minute order options, and its objective is to minimize the total machines' installation cost, transportation cost, subcontracting cost, and the expected (second-stage) transportation, inventory, and penalty costs associated with the last minute ordering after a realization of uncertain demand. We generate the aforementioned cost parameters as follows: the subcontract cost is 4 per unit, i.e., $g_{i}=4$ for all $i$, the inventory cost is 0.35 per unit, i.e., $w_{i n v}^{i}=0.35$, for all $i$, the last minute order cost is 3 times the trans- 
portation cost $t_{i j}$, i.e., $w_{s c}=3$, and the demand penalty is set to 100 per unit, i.e., $w_{\text {pen }}=100$. The capacity of modules and their installation costs, $\left(\alpha_{k}, c_{i}^{k}\right)$, belong to the set $\{(1000,2700),(4000,9000),(10000,18000),(16000,25000)\}$. We allow at most 200 subcontract items, i.e., $r=2000$, and the maximum number of modules at each facility is at most 4, i.e., $s_{i}=4$ for $i \in P$.

\subsection{Generation of structured TSS-CMIP test instances}

For our computational experiments, we consider the extensive formulation of reformulated TSS-CMIP with $p=1$ or $p=2$, and structured CMIPs in the second stage (as discussed in previous section), i.e. either $E_{\omega}^{j}=\mathbf{I}$ (an identity matrix) or $E_{\omega}^{j}$ is a randomly generated deterministic network flow matrix for $j \in J$, and $W_{\omega}$ is a deterministic network flow matrix for all $\omega \in \Omega$. Specifically, we generate network flow matrices as follows. First, we randomly generate two graph edge vectors $v_{1}, v_{2} \in \mathbb{Z}_{+}^{m^{\prime}}$, where $m^{\prime}$ is the number of rows of desired matrix. For any element in $v_{1}$ and $v_{2}$, i.e., $v_{1}^{i}$ and $v_{2}^{i}$ for $1 \leq i \leq m^{\prime}$, we have $v_{1}^{i}, v_{2}^{i} \leq$ $n^{\prime}$ and $v_{1}^{i} \neq v_{2}^{i}$, where $n^{\prime}$ is the number of columns of desired matrix. Here, $v_{1}$ and $v_{2}$ are vectors of index of graph nodes. We create an undirected graph with these $m^{\prime}$ node pairs using MATLAB function $g r a p h\left(v_{1}, v_{2}\right)$. This function specifies $\left(v_{1}^{i}, v_{2}^{i}\right)$ for $1 \leq$ $i \leq m^{\prime}$ as an edge, and generates a graph with $m^{\prime}$ edges and $\hat{n}\left(\hat{n} \leq n^{\prime}\right)$ nodes, where $\hat{n}=\max \left\{v_{1}^{1}, \ldots, v_{1}^{m^{\prime}}, v_{2}^{1}, \ldots, v_{2}^{m^{\prime}}\right\}$. We obtain the network flow matrix of size $m^{\prime} \times \hat{n}$ corresponding to this graph and set the remaining $n^{\prime}-\hat{n}$ columns to $\mathbf{0}$, thereby providing a network flow matrix of desired size. For each structured TSS-CMIP with $p=2$ and only integer variables in the second stage, we generate two sets of random instances: instances from the first problem set are motivated from the Stochastic Integer Programming Library (SIPLIB) TSS-MILP instances [2], in particular stochastic server location problem (SSLP) and stochastic multiple binary knapsack problem (SMBKP) instances, whereas for the second problem set, we consider instances with larger number of scenarios (up to 10,000). More specifically, in the first problem set, we generate random instances with similar problem size as of SSLP and SMBKP instances but with uncertain cost-coefficients, technology matrix, $F_{\omega}^{j}, h_{\omega}^{j}$ and right-hand-side.

Likewise for TSS-CMIPs with $p=1$, we consider instances with network flow $E_{\omega}^{j}$ matrix for all $j \in J$ and larger number of scenarios (up to 50,000). In Tables 1, 2, and 3, we provide details of problem categories for different types of structured second-stage CMIPs, i.e., $E_{\omega}^{j}$ is an identity matrix or any network flow matrix, used for our experiments. We denote the number of linear constraints and number of integer variables by \#LCon and \#IVar, respectively, in each stage. We use \#PCCon and \# $p$-OCcon to denote the number of polyhedral conic constraints (23) or number of rows in $E_{\omega}^{j}$, and number of $p$-order conic constraints (24), respectively, in the second stage.

We use SCMIP. $\alpha . \beta . \lambda$ to denote our instance category in Tables 1,2 , and 3 , where $\alpha$ is the number of integer variables in the first stage, $\beta$ is the number of integer variables in the second stage, and $\lambda$ is the number of $p$-order conic constraints in the second stage. Note that in Table 2, \#PCCon is the same as \#IVar in the second stage as $E_{\omega}^{j}$ is an identity matrix. For each instance category, we generate instances as follow: we draw $c$ from uniform $[0,10], A, b$ from uniform $[0,100], r_{\omega}, T_{\omega}$ from integer uniform $[-100,100], F_{\omega}^{j}$ from integer uniform $[-10,10], h_{\omega}^{j}$ from uniform $[-10,10]$. Moreover, for instance categories in Table 2, we let $g_{\omega}=\mathbf{0}$ and $\hat{g}_{\omega}^{j}=1$ for $\omega \in \Omega, j \in J$, while for instance categories in Tables 1 and $3, g_{\omega}$ is randomly generated from uniform $[-1,1]$ and $\hat{g}_{\omega}^{j}$ is randomly gen- 
Table 1 Details of TSS-CMIP Instances with $p=1$ and $E_{\omega}^{j}$ is network flow matrix

\begin{tabular}{|c|c|c|c|c|c|c|}
\hline \multirow{2}{*}{$\begin{array}{l}\text { Instance } \\
\text { Category }\end{array}$} & \multicolumn{2}{|l|}{ Stage I } & \multicolumn{4}{|l|}{ Stage II } \\
\hline & \#LCon & \#IVar & \#LCon & \#IVar & \#PCCon & \#p-OCon \\
\hline SCMIP.25.50.5 & 10 & 25 & 20 & 50 & 25 & 5 \\
\hline SCMIP.50.100.5 & 25 & 50 & 40 & 100 & 50 & 5 \\
\hline SCMIP.100.150.5 & 75 & 100 & 50 & 150 & 70 & 5 \\
\hline SCMIP.5.10.5 & 50 & 5 & 2 & 10 & 3 & 5 \\
\hline
\end{tabular}

Table 2 Details of TSS-CMIP Instances with $p=2$ and $E_{\omega}^{j}=\mathbf{I}$

\begin{tabular}{|c|c|c|c|c|c|c|c|}
\hline \multirow{2}{*}{$\begin{array}{l}\text { Problem } \\
\text { Set }\end{array}$} & \multirow{2}{*}{$\begin{array}{l}\text { Instance } \\
\text { Category }\end{array}$} & \multicolumn{2}{|l|}{ Stage I } & \multicolumn{4}{|l|}{ Stage II } \\
\hline & & \#LCon & \#IVar & \#LCon & \#IVar & \#PCCon & $\# p$-OCon \\
\hline \multirow[t]{5}{*}{ I } & SCMIP.5.125.1 & 1 & 5 & 30 & 125 & 125 & 1 \\
\hline & SCMIP.10.500.1 & 1 & 10 & 60 & 500 & 500 & 1 \\
\hline & SCMIP.15.675.1 & 1 & 15 & 60 & 675 & 675 & 1 \\
\hline & SCMIP.20.800.1 & 1 & 20 & 60 & 800 & 800 & 1 \\
\hline & SCMIP.240.120.1 & 50 & 240 & 5 & 120 & 120 & 1 \\
\hline \multirow[t]{2}{*}{ II } & SCMIP.5.10.1 & 5 & 5 & 5 & 10 & 10 & 1 \\
\hline & SCMIP.10.10.1 & 5 & 10 & 10 & 10 & 10 & 1 \\
\hline
\end{tabular}

Table 3 Details of TSS-CMIP Instances with $p=2$ and $E_{\omega}^{j}$ is network flow matrix

\begin{tabular}{|c|c|c|c|c|c|c|c|}
\hline \multirow{2}{*}{$\begin{array}{l}\text { Problem } \\
\text { Set }\end{array}$} & \multirow{2}{*}{$\begin{array}{l}\text { Instance } \\
\text { Category }\end{array}$} & \multicolumn{2}{|l|}{ Stage I } & \multicolumn{4}{|l|}{ Stage II } \\
\hline & & \#LCon & \#IVar & \#LCon & \#IVar & \#PCCon & \#p-OCon \\
\hline \multirow[t]{4}{*}{ I } & SCMIP.5.125.3 & 1 & 5 & 30 & 125 & 100 & 3 \\
\hline & SCMIP.10.500.3 & 1 & 10 & 60 & 500 & 300 & 3 \\
\hline & SCMIP.15.675.3 & 1 & 15 & 60 & 675 & 400 & 3 \\
\hline & SCMIP.240.120.3 & 50 & 240 & 5 & 120 & 100 & 3 \\
\hline \multirow[t]{5}{*}{ II } & SCMIP.10.25.3 & 5 & 10 & 10 & 25 & 10 & 3 \\
\hline & SCMIP.10.50.3 & 5 & 10 & 20 & 50 & 25 & 3 \\
\hline & SCMIP.10.75.3 & 5 & 10 & 20 & 75 & 35 & 3 \\
\hline & SCMIP.25.50.3 & 10 & 25 & 20 & 50 & 25 & 3 \\
\hline & SCMIP.25.100.3 & 10 & 25 & 20 & 100 & 80 & 3 \\
\hline
\end{tabular}

erated from uniform $[-10,10]$. Since $y_{\omega}^{j}$ is an unrestricted variable and $g_{\omega} \in[-1,1]$ for $j \in J$ and $\omega \in \Omega$, we observed that many randomly generated TSS-CMIP instances have unbounded solution values. Therefore, in order to obtain finite optimal solution values, we impose an upper bound of 100 and a lower bound of -100 on each $y_{\omega}^{j}$ variable. Note that when $E_{\omega}^{j}=\mathbf{I}$, the number of integer variables in the second stage, i.e., $q$, is same as number of polyhedral conic constraints, i.e., $m_{2}$. Whereas for instance categories in Table 1 and 3 , $E_{\omega}^{j}$ is not a square matrix. These instances will be available at computational Operations Research exchange (cORe) https://core.isrd.isi.edu/ and GitHub page of the authors. 


\subsection{Computational framework}

In this section, we evaluate the effectiveness of our scenario-based cuts by performing computational experiments on instances belonging to the aforementioned instance categories with different number of scenarios. The results of our experiments are presented in Tables 4, 6, 7, and 9. Each row in Tables 6 and 7 reports the average over five randomly generated instances corresponding to the instance category in Table 1 and 2, respectively, while each row in Tables 4 and 9 report the average over three randomly generated (relatively harder) instances belonging to MM-SFLP-S and instance category in Table 3, respectively. For instances in Table 1, we perform three experiments: NO-SCUT, WITH-SCUTS, and BD-WITH-SCUTS. While for instances in Table 2, 3 and 4, we only perform the first two experiments. In NO-SCUT, we solve the reformulated extensive formulation of the problem using CPLEX 12.70 with its default settings, without adding our scenario-based cuts. (In order to improve the performance of CPLEX, we substitute unrestricted variable $y_{\omega}^{j}$ by $y_{\omega}^{j+}-y_{\omega}^{j-}$ in our experiments, where $y_{\omega}^{j+}, y_{\omega}^{j-} \geq 0$ for $j \in J, \omega \in \Omega$.) Whereas in WITH-SCUTS, we add our scenario-based linear cuts (provided in Theorem 7 or derived through reformulation of $\overline{\mathcal{P}}_{p c h}$ in Corollary 4 for $\Gamma=\Omega$ ), a priori to the reformulated extensive formulation of the problem instance, relax the integrality constraints of second stage integer variables, and use CPLEX 12.70 with its default settings to solve it. In BD-WITH-SCUTS for TSS-CMIP with $p=1$, we first convexify the second stage problem by adding our parametric cuts (provided in Theorem 7), and then solve it using Benders' decomposition routine of CPLEX 12.70. For NO-SCUT and WITH-SCUTS, we allow presolve option in CPLEX 12.70; while for BD-WITH-SCUTS, we turn it off. All experiments are performed on a 8-core Xeon $2.4 \mathrm{GHz}$ machine with 24 GB RAM running with Windows 10 .

In Tables 4, 5, 6, 7 and 9, we report following statistics: the number of integer variables in the extensive formulation with(out) scenario-based cuts (\#IVar), the number of linear constraints in the extensive formulation without our scenario-based cuts (\#LCon), and the number of linear scenario-based cuts added in the the extensive formulation (\#LCuts). Also, we denote the total time taken to solve TSS-CMIP and TSDR-CMIP instances without and with our scenario-based cuts in extensive formulation by T-EF and T-EFC, respectively, and the percentage of integrality gap closed by a priori addition of scenario-based cuts by ImprG\% $=100 \times\left(V_{p c u t}-V_{c p}\right) /\left(V_{\text {mip }}-V_{c p}\right)$, where $V_{p c u t}, V_{c p}$, and $V_{\text {mip }}$ denote the optimal objective value of continuous relaxation of extensive formulation with our scenariobased cuts, continuous relaxation of extensive formulation without our scenario-based cuts, and original formulation, respectively. In Table 6, T-BDC denotes the time taken to solve TSS-CMIP with $p=1$ and linear programming equivalent of the second stage CMIP, using Benders' decomposition routine of CPLEX. We use TL to notify that CPLEX cannot solve the corresponding instance within 3 hours time limit and OM to notify that our system ran out of 24 GB memory when solving this instance. Instances for which we could not obtain the optimal value due to TL or OM, we put - in column ImprG\%.

\subsubsection{Computational results for MM-SFLP-S and MM-DRFLP-S instances.}

In Table 4, we observe that by adding the scenario-based conic MIR cuts a priori, the number of integer variables (\#IVar) is significantly reduced for MM-SFLP-S instances. The average gap closed by the scenario-based cuts is $98 \%$. Without the scenario-based cuts, only 8 out of 24 MM-SFLP-S instances can be solved by CPLEX within the time limit. In contrast, after adding the cuts, 10 out of 16 foregoing unsolved instances are solved in 2576 seconds (on 
Table 4 Results of computational experiments for the MM-SFLP-S instances

\begin{tabular}{|c|c|c|c|c|c|c|c|c|}
\hline \multirow{2}{*}{$\begin{array}{l}\text { Instance } \\
\text { Category }\end{array}$} & \multirow[t]{2}{*}{$|\Omega|$} & \multicolumn{3}{|c|}{ NO-SCUT } & \multicolumn{4}{|c|}{ WITH-SCUTs } \\
\hline & & \#IVar & \#LCon & $\mathrm{T}-\mathrm{EF}$ & \#IVar & \#LCuts & T-EFC & ImprG\% \\
\hline & 100 & 80944 & 11633 & 72.8 & 944 & 5000 & 52 & 98 \\
\hline$\alpha_{1}=1000$ & 500 & 400944 & 58033 & 1173 & 944 & 25000 & 885 & 98 \\
\hline \multirow[t]{3}{*}{$\alpha_{2}=4000$} & 750 & 600944 & 87033 & TL & 944 & 37500 & 2125 & 98 \\
\hline & 1000 & 800944 & 116033 & 4913 & 944 & 50000 & 3633 & 98 \\
\hline & 100 & 80944 & 11633 & $\mathrm{TL}$ & 944 & 5000 & 145.1 & 89 \\
\hline$\alpha_{1}=1000$ & 500 & 400944 & 58033 & $\mathrm{TL}$ & 944 & 25000 & 3513 & 90 \\
\hline \multirow[t]{2}{*}{$\alpha_{2}=16000$} & 750 & 600944 & 87033 & $\mathrm{TL}$ & 944 & 37500 & $\mathrm{TL}$ & 90 \\
\hline & 1000 & 800944 & 116033 & $\mathrm{TL}$ & 944 & 50000 & $\mathrm{TL}$ & - \\
\hline$\overline{\alpha_{1}}=1000$ & 100 & 81072 & 11633 & TL & 1072 & 5000 & 360 & 92 \\
\hline$\alpha_{2}=4000$ & 500 & 401072 & 58033 & 6329 & 1072 & 25000 & 8645 & 92 \\
\hline$\alpha_{3}=10000$ & 750 & 601072 & 87033 & $\mathrm{TL}$ & 1072 & 37500 & $\mathrm{TL}$ & - \\
\hline$\alpha_{4}=16000$ & 1000 & 801072 & 116033 & $\mathrm{TL}$ & 1072 & 50000 & $\mathrm{TL}$ & - \\
\hline
\end{tabular}

Table 5 Results of computational experiments for the MM-DRFLP-S instances

\begin{tabular}{|c|c|c|c|c|c|c|}
\hline \multirow{2}{*}{$\begin{array}{l}\text { Instance } \\
\text { Category }\end{array}$} & \multirow[t]{2}{*}{$|\Omega|$} & \multirow[t]{2}{*}{$|\mathfrak{P}|$} & \multirow{2}{*}{$\begin{array}{l}\text { NO-SCUT } \\
\text { T-EF }\end{array}$} & \multicolumn{3}{|c|}{ WITH-SCUTs } \\
\hline & & & & \#LCuts & T-EFC & ImprG\% \\
\hline & 100 & 5 & $\mathrm{TL}$ & 5000 & 180 & 98.1 \\
\hline$\alpha_{1}=1000$ & 100 & 10 & $\mathrm{TL}$ & 5000 & 235 & 97.9 \\
\hline \multirow[t]{3}{*}{$\alpha_{2}=4000$} & 100 & 25 & $\mathrm{TL}$ & 5000 & 474 & 98.1 \\
\hline & 100 & 50 & $\mathrm{TL}$ & 5000 & 904 & 97.9 \\
\hline & 100 & 5 & TL & 5000 & 777 & 90.1 \\
\hline$\alpha_{1}=1000$ & 100 & 10 & $\mathrm{TL}$ & 5000 & 744 & 90.1 \\
\hline \multirow[t]{2}{*}{$\alpha_{2}=16000$} & 100 & 25 & $\mathrm{TL}$ & 5000 & 959 & 89.9 \\
\hline & 100 & 50 & $\mathrm{TL}$ & 5000 & 1666 & 89.9 \\
\hline$\alpha_{1}=1000$ & 100 & 5 & $\mathrm{TL}$ & 5000 & 1601 & 92.2 \\
\hline$\alpha_{2}=4000$ & 100 & 10 & $\mathrm{TL}$ & 5000 & 1944 & 91.9 \\
\hline$\alpha_{3}=10000$ & 100 & 25 & $\mathrm{TL}$ & 5000 & 1252 & 91.7 \\
\hline$\alpha_{4}=16000$ & 100 & 50 & $\mathrm{TL}$ & 5000 & 3008 & 91.9 \\
\hline
\end{tabular}

average). After adding the scenario-based cuts, CPLEX took lesser time to solve 6 out of above 8 instances, i.e., 1681 seconds on average. Whereas it took 2201 seconds (on average) for CPLEX to solve these instances without the cuts.

In Table 5, we observe that by adding scenario-based conic MIR cuts a priori to the extensive formulation of MM-DRFLP-S, the average gap closed is $93.3 \%$. Without these cuts, CPLEX could not solve any of the 36 MM-DRFLP-S instances within the time limit. In contrast, after adding the cuts, all these instances can be solved in 1145 seconds (on average). Recall that MM-SFLP-S is equivalent to MM-DRFLP-S with $|\mathfrak{P}|=1$, therefore as expected, the time taken to solve MM-DRFLP-S instances in Table 5 is more than the time taken to solve MM-SFLP-S instances in Table 4 with $|\Omega|=100$. 


\subsubsection{Computational results for TSS-CMIPs where $p=1$ and $E_{\omega}^{j}$ are network flow matrices for all $j \in J$.}

In Table 6, we observe that by adding scenario-based cuts a priori, the number of integer variables (\#IVar) is significantly reduced. Without our scenario-based cuts, CPLEX with its default settings took 800 seconds (on average) to solve the extensive formulation of the TSS-CMIP instances where $p=1$ and $E_{\omega}^{j}, j \in J$, are network flow matrices. However, by adding our cuts, CPLEX took 143 seconds (on average) to solve the extensive formulation of these instances, and reduced the time by up to 25 times and 4.6 times (on average). Our scenario-based cuts closed the integrality gap by almost $100 \%$. It is worth noting that even though we disabled presolve when using Benders decomposition routine, for instance category SCMIP.5.10.5, T-BDC is on average $79.83 \%$ of T-EF.

\subsubsection{Results for TSS-CMIPs and TSDR-CMIPs with $p=2$ and $E_{\omega}^{j}=\mathrm{I}$.}

For TSS-CMIP and TSDR-CMIPs with $p=2$, adding our scenario-based cuts in the extensive formulation and relaxing integrality restrictions on second stage integer variables provide an approximation of the problem. Let the approximation ratio be defined by $R \%=100 \times V_{\text {approx }} / V_{\text {mip }}$ where $V_{\text {approx }}$ is the optimal objective value obtained from the experiment WITH-SCUTS (after adding scenario-based cuts in the extensive formulation and relaxing the integrality constraints on the second stage variables). We observe that the approximation ratio of all instances, with known $V_{\text {mip }}$, in Table 7 is greater than $99.99 \%$, thereby demonstrating the strength of our scenario-based cuts. In other words, these cuts provide near-optimal solution for TSS-CMIPs with $p=2$ and $E_{\omega}^{j}=\mathbf{I}$.

Now by comparing T-EF and T-EFC in Table 7, we observe that adding our scenariobased linear cuts (a priori) significantly reduces the time taken to solve the reformulated extensive formulation of the TSS-CMIP instances, using CPLEX with its default settings. More specifically, after adding our scenario-based cuts, CPLEX solved 132 out of 135 randomly generated instances within the time limit, except three instances of SCMIP.10.50.1 with 500 scenarios. Whereas, without our cuts, CPLEX could not solve 35 out of 135 TSSCMIP instances within 3 hours time limit ( 32 instances) and allocated memory ( 3 instances), and took 148 seconds (on average) to solve the remaining 100 instances in comparison to 32 seconds (on average) after adding our cuts. Additionally, WITH-SCUTS took 22 minutes (on average) for 32 out of 35 (unsolvable) instances. Overall, for instances in Table 7, our scenario-based cuts closed the integrality gap by $19.1 \%$ (on average) for instances solved to optimality using NO-SCUT, and reduced the time taken to solve the TSS-CMIP instances by 5 times (on average).

In particular, for problem set I, WITH-SCUTS were performed at least 2 times and up to 19 times faster than NO-SCUT. Whereas for problem set II, WITH-SCUTS is 5 times (on average) and up to 39 times faster than NO-SCUT. It is interesting to observe that while by adding our scenario-based cuts, there is a trade-off between the decrease in the number of integer variables and increase in the number of constraints. For instances such as SCMIP 10.10 .1 where $|\Omega| \in\{50,100,500,1000\}$, WITH-SCUTS took marginally longer time that NO-SCUT. However, for instances with larger number of scenarios, WITH-SCUTS are notably faster than NO-SCUT.

In Table 8, we present results for distributionally robust versions of TSS-CMIPs instances with $p=2$ and $E_{\omega}^{j}=\mathbf{I}$. Specifically, we consider three instance categories from Table 7 that were solved in a reasonable time by CPLEX even without adding scenario-based cuts and for 


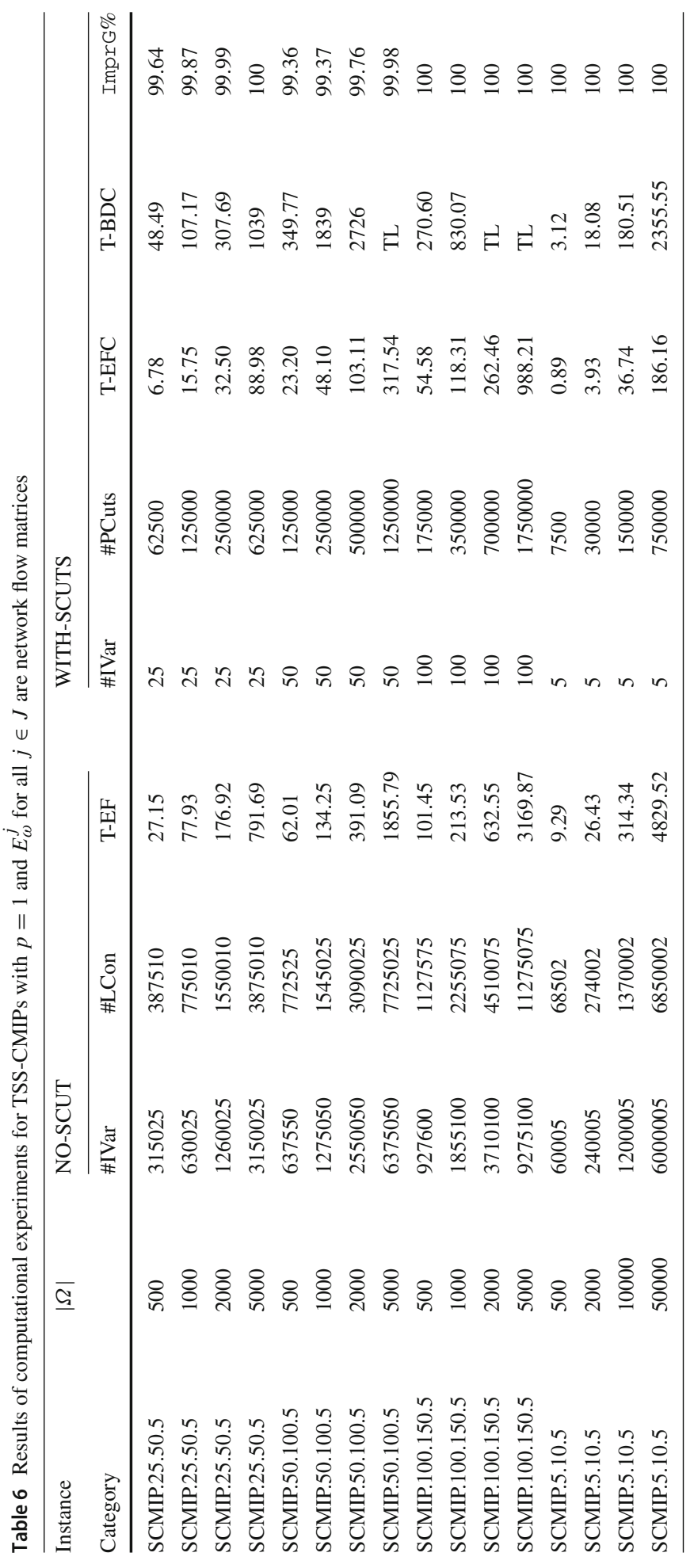




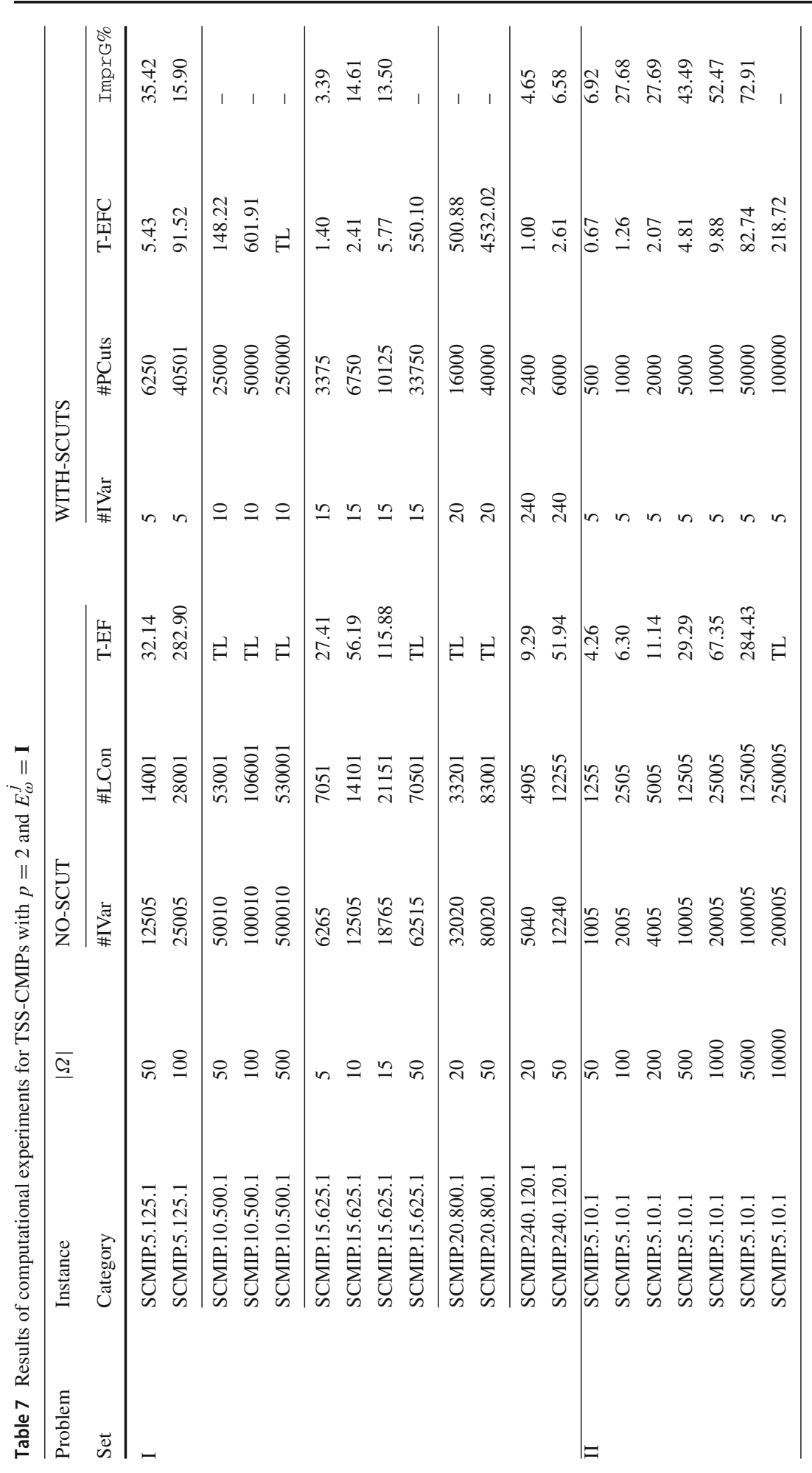




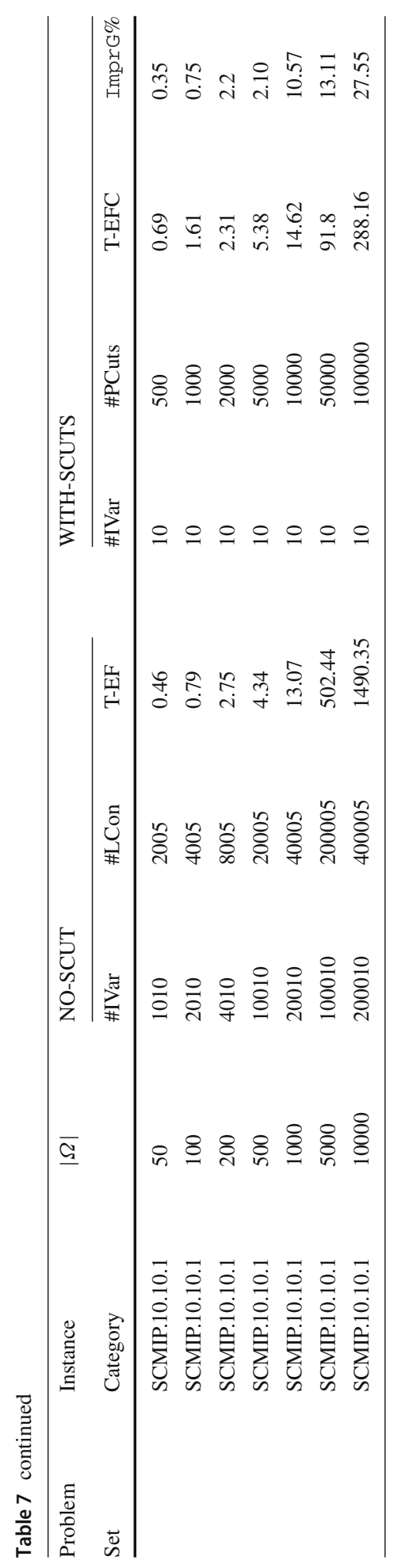

照 Springer 


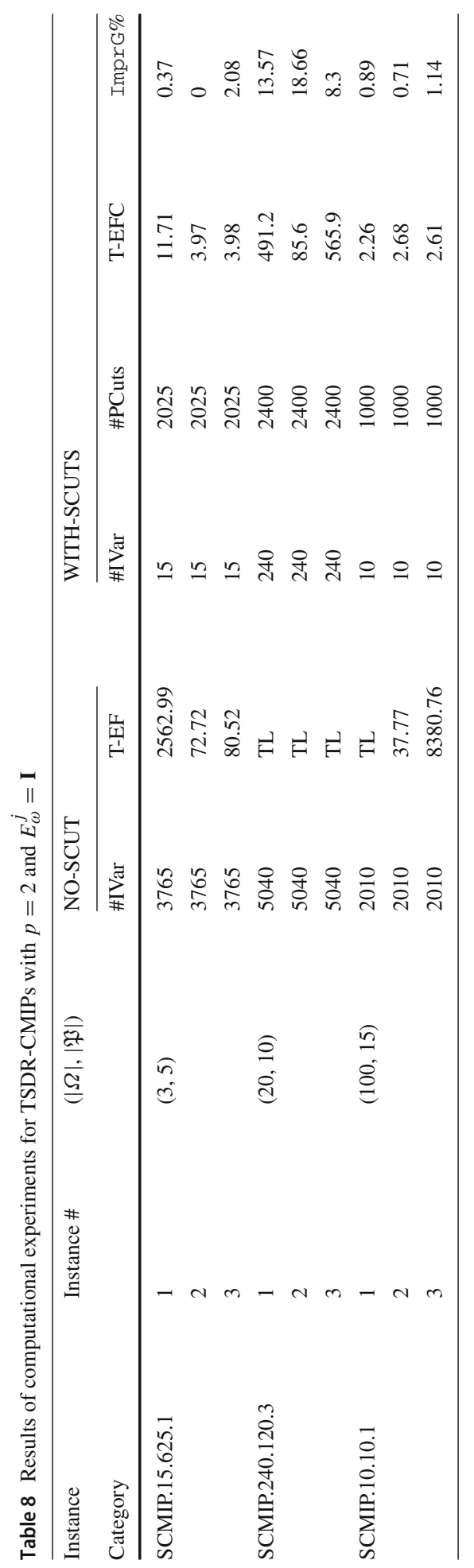




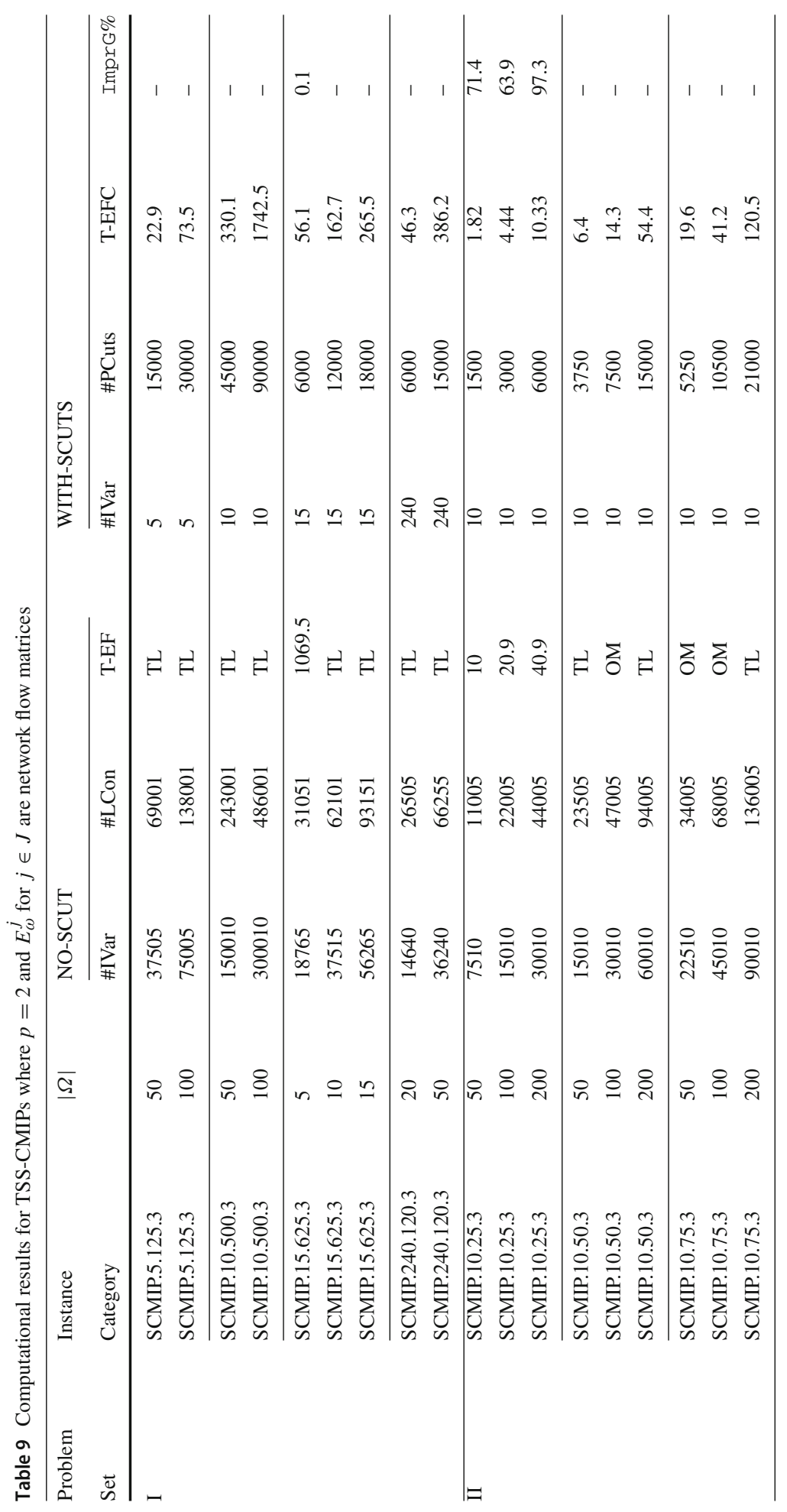




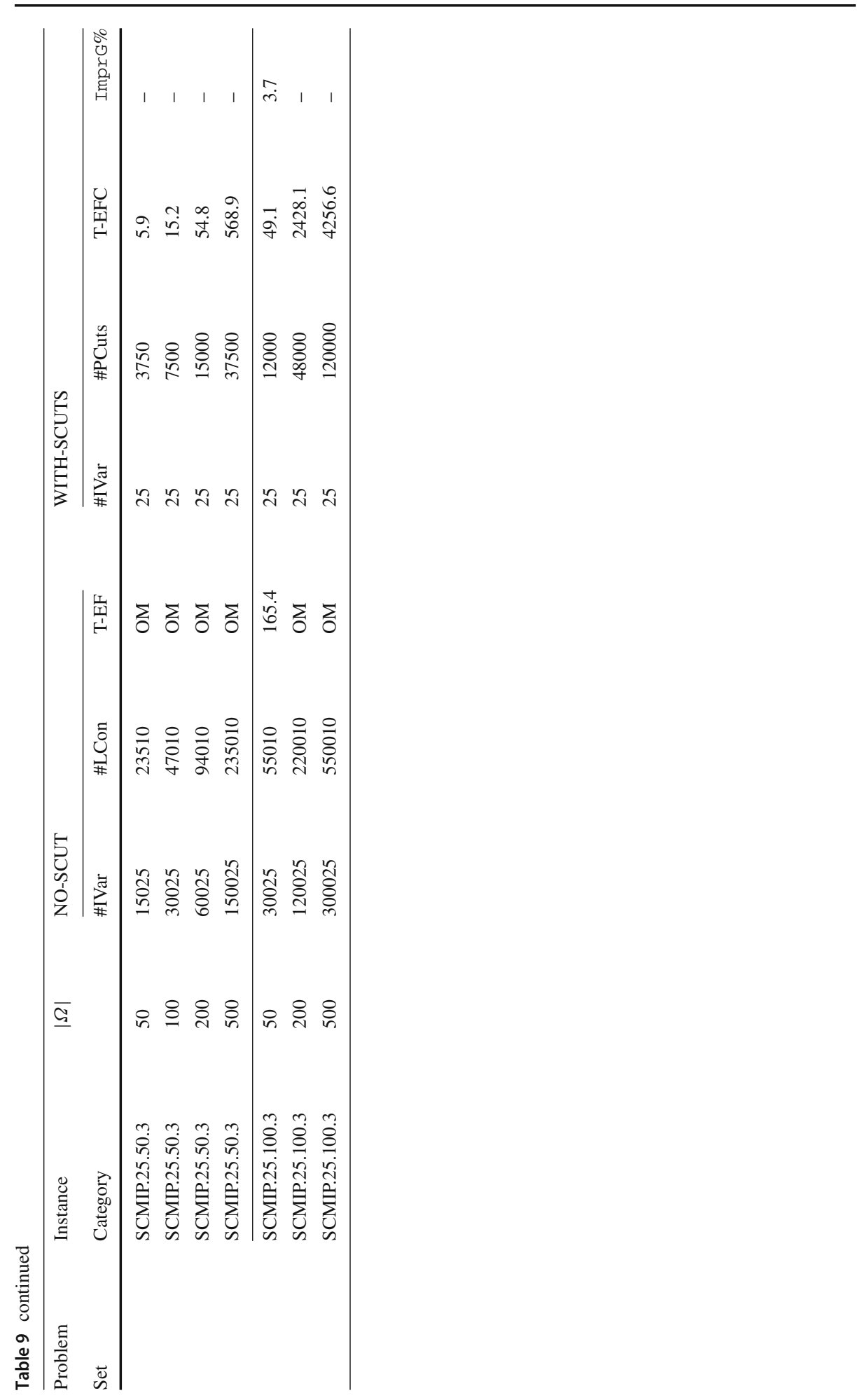


$|\mathfrak{P}|=1$. We generated three TSDR-CMIP instances for each category, i.e., SCMIP.15.625.1, SCMIP.240.120.3, and SCMIP.10.10.1, with fixed number of scenarios $|\Omega|$ and distributions in the ambiguity set $|\mathfrak{P}|$. We observe that CPLEX could not solve the reformulated extensive formulation of 4 out of 9 instances within a time limit of 3 hours. However, after adding the scenario-based linear cuts (a priori), CPLEX solved these instances within 286 seconds (on average). For the remaining instances, by comparing T-EF and T-EFC in Table 8, we again observe that adding the scenario-based linear cuts reduces the time taken to solve the instances by 446 times (on average).

\subsubsection{Computational results for TSS-CMIPs with $p=2$ where $E_{\omega}^{j}$ for $j \in J$ are network flow matrix.}

The problem instances considered in Table 9 are much harder to solve than problem instances considered in Table 7, primarily because of multiple conic constraints (24) corresponding to each scenario (as $|J|=3$ ) and multiple integer variables in each constraint (23). Nonetheless, we again observe that the approximation ratio $\mathrm{R} \%$ for all problem instances, with known $V_{\text {mip }}$, in Table 9 is greater than 99.99\%. It is evident from column T-EF in Table 9 which shows that CPLEX 12.70 with its default settings could not solve reformulated extensive formulation of 50 out of 75 TSS-CMIP instances (without our scenario-based cuts) within a time limit of 3 hours and the allocated 24 GB RAM. In contrast, after adding our scenariobased cuts, we solved all 75 instances in 449.64 seconds (on average). For instances solved to optimality using NO-SCUT, our cuts closed the integrality gap by $47.29 \%$ (on average) and reduced the time taken to solve the TSS-CMIP instances by 10.32 times (on average). It is worth to note that even though ImprG\% is small for some instances (i.e., SCMIP.15.625.3, SCMIP.240.120.3, and SCMIP.25.100.3 for $|\Omega| \in\{50,200\})$, CPLEX with its default settings (without scenario-based cuts) took longer time, at least 2.4 times and up to 18 times, to solve these instances, in comparison to solving them using CPLEX with our scenario-based cuts.

\section{Conclusion}

We presented conditions under which the addition of scenario-based nonlinear cuts in the extensive formulation of TSS-CMIPs is sufficient to relax the integrality restrictions on the second stage integer variables without impacting the integrality of the optimal solution of the TSS-CMIP. We introduced structured TSS-CMIPs and cuts for them which satisfy these conditions, thereby providing partial convex hull for them. We introduced TSS-CMIPs and TSDR-CMIPs with structured $p$-order CMIPs in the second stage, derived scenario-based conic MIR cuts for them, and proved that these cuts provide conic/linear programming equivalent or approximation for the second stage CMIPs with $p=1$ or $p \geq 2$, respectively. We also introduced a multi-module capacitated stochastic facility location problem with subcontracting (MM-SFLP-S) and demonstrated the applicability of the foregoing results for solving this problem. We also computationally evaluated the effectiveness of the scenario-based cuts by considering instances of MM-SFLP-S, its distributionally robust variant, and structured TSS-CMIPs with polyhedral CMIPs and second-order CMIPs in the second stage, i.e. $p=1$ and $p=2$, respectively. Our computational results showed that adding scenario-based cuts to the extensive formulation significantly reduced the time taken to solve extensive formulation of TSS-CMIPs and TSDR-CMIPs compared to solving the same problem instances without 
these cuts, using CPLEX 12.70 with its default settings. Furthermore, we derived (partial) convex hull for new deterministic multi-constraint polyhedral conic mixed integer sets with multiple integer variables.

Acknowledgements This research is funded by National Science Foundation Grant CMMI- 1824897 and Junior Faculty Award of ICTAS at Virginia Tech, which are gratefully acknowledged.

\section{Appendix A: Proof of Theorem 5}

Given $\omega_{1} \in \Omega$, let

$$
\operatorname{conv}\left(\overline{\mathcal{K}}_{\omega_{1}}(x)\right)=\overline{\mathcal{K}}_{\text {tight }}^{\omega_{1}}(x)
$$

for all $x \in X$. Suppose that a point $\left(\hat{x}, \hat{y}_{\omega_{1}}, \ldots, \hat{y}_{\omega_{|\Omega|}}, \hat{d}_{\omega_{1}, 0}, \ldots, \hat{d}_{\omega_{|\Omega|}, 0}\right) \in \overline{\mathcal{P}}$, which implies $\left(\hat{y}_{\omega_{1}}, \hat{d}_{\omega_{1}, 0}\right) \in \overline{\mathcal{K}}_{\omega_{1}}(\hat{x})$ and because of assumption (75), $\left(\hat{y}_{\omega_{1}}, \hat{d}_{\omega_{1}, 0}\right) \in \overline{\mathcal{K}}_{\text {tight }}^{\omega_{1}}(\hat{x})$. Since $\left(\hat{x}, \hat{y}_{\omega_{1}}, \ldots, \hat{y}_{\omega_{|\Omega|}}, \hat{d}_{\omega_{1}, 0}, \ldots, \hat{d}_{\omega_{|\Omega|}, 0}\right)$ satisfies all defining constraints of $\overline{\mathcal{P}}_{p c h, 1}$, defined by

$$
\begin{array}{lr}
T_{\omega} x+W_{\omega} y_{\omega} \geq r_{\omega}, & \omega \in \Omega, \\
\left\|E_{\omega}^{j} y_{\omega}^{j}+F_{\omega}^{j} x-h_{\omega}^{j}\right\|_{p} \leq d_{\omega, 0}^{j}, & j \in J, \omega \in \Omega, \\
\left\|\bar{E}_{\omega_{1}, l}^{j} y_{\omega_{1}}^{j}+\bar{F}_{\omega_{1}, l}^{j} x-\bar{h}_{\omega_{1}, l}^{j}\right\|_{p} \leq d_{\omega_{1}, 0}^{j}, & l \in \mathcal{L}, j \in J, \\
x \in X, d \in \mathbb{R}_{+}^{|J| \times|\Omega|}, y_{\omega_{1}}^{j} \in \mathbb{R}^{q}, & j \in J, \\
y_{\omega}^{j} \in \mathbb{Z}^{q}, & j \in J, \omega \in \Omega \backslash\left\{\omega_{1}\right\},
\end{array}
$$

the point $\left(\hat{x}, \hat{y}_{\omega_{1}}, \ldots, \hat{y}_{\omega_{|S|} \mid}, \hat{d}_{\omega_{1}, 0}, \ldots, \hat{d}_{\omega_{|\Omega|}, 0}\right) \in \overline{\mathcal{P}}_{p c h, 1}$. Therefore,

$$
\overline{\mathcal{P}} \subseteq \overline{\mathcal{P}}_{p c h, 1} \text { and } \operatorname{conv}(\overline{\mathcal{P}}) \subseteq \operatorname{conv}\left(\overline{\mathcal{P}}_{p c h, 1}\right)
$$

Now suppose that a point $\left(\hat{x}, \hat{y}_{\omega_{1}}, \ldots, \hat{y}_{\omega_{|\Omega|} \mid}, \hat{d}_{\omega_{1}, 0}, \ldots, \hat{d}_{\omega_{|\Omega|}, 0}\right) \in \overline{\mathcal{P}}_{p c h, 1}$. Then point $\left(\hat{y}_{\omega_{1}}, \hat{d}_{\omega_{1}, 0}\right) \in \overline{\mathcal{K}}_{\text {tight }}^{\omega_{1}}(\hat{x})$. Also, because of assumption (75), $\left(\hat{y}_{\omega_{1}}, \hat{d}_{\omega_{1}, 0}\right) \in \operatorname{conv}\left(\overline{\mathcal{K}}_{\omega_{1}}(\hat{x})\right)$, and hence this point can be written as convex combination of finite number of points, $\bar{\eta}_{\omega_{1}}^{k} \in$ $\mathbb{R}^{q+|J|}$ for $k \in\{1,2, \ldots\}$, belonging to $\overline{\mathcal{K}}_{\omega_{1}}(\hat{x})$, i.e.,

$$
\left(\hat{y}_{\omega_{1}}, \hat{d}_{\omega_{1}, 0}\right)=\sum_{k} \lambda_{k} \bar{\eta}_{\omega_{1}}^{k}
$$

where $\sum_{k} \lambda_{k}=1$ and $\lambda_{k} \geq 0$ for all $k$. Since $\left(\hat{y}_{\omega}, \hat{d}_{\omega, 0}\right) \in \overline{\mathcal{K}}_{\omega}(\hat{x})$ for $\omega \in \Omega \backslash\left\{\omega_{1}\right\}$ and $\bar{\eta}_{\omega_{1}}^{k} \in \overline{\mathcal{K}}_{\omega_{1}}(\hat{x})$,

$$
\left(\hat{x}, \bar{\eta}_{\omega_{1}}^{k}, \hat{y}_{\omega_{2}}, \hat{d}_{\omega_{2}, 0}, \ldots, \hat{y}_{\omega_{|\Omega|} \mid}, \hat{d}_{\omega_{|\Omega|, 0}}\right) \in \overline{\mathcal{P}}
$$

for all $k$ as $\overline{\mathcal{K}}_{\omega}(\hat{x})=\operatorname{Proj}_{x=\hat{x}, y_{\omega}, d_{\omega}}(\overline{\mathcal{P}})$ for all $\omega \in \Omega$. This implies

$$
\left(\hat{x}, \sum_{k} \lambda_{k} \bar{\eta}_{\omega_{1}}^{k}=\left(\hat{y}_{\omega_{1}}, \hat{d}_{\omega_{1}, 0}\right), \hat{y}_{\omega_{2}}, \hat{d}_{\omega_{2}, 0}, \ldots, \hat{y}_{\omega_{|S|} \mid}, \hat{d}_{\omega_{|\Omega|} \mid, 0}\right) \in \operatorname{conv}(\overline{\mathcal{P}}) .
$$


Hence,

$$
\overline{\mathcal{P}}_{p c h, 1} \subseteq \operatorname{conv}(\overline{\mathcal{P}}) \text { and } \operatorname{conv}\left(\overline{\mathcal{P}}_{p c h, 1}\right) \subseteq \operatorname{conv}(\overline{\mathcal{P}})
$$

From (76) and (77), we get

$$
\overline{\mathcal{P}} \subseteq \overline{\mathcal{P}}_{p c h, 1} \subseteq \operatorname{conv}(\overline{\mathcal{P}})=\operatorname{conv}\left(\overline{\mathcal{P}}_{p c h, 1}\right)
$$

which means $\overline{\mathcal{P}}_{p c h, 1}$ is a partial convex hull of $\overline{\mathcal{P}}$.

Next, we assume that $\operatorname{conv}\left(\overline{\mathcal{K}}_{\omega_{2}}(x)\right)=\overline{\mathcal{K}}_{\text {tight }}^{\omega_{2}}(x)$ for all $x \in X$. Replacing $\overline{\mathcal{P}}$ by $\overline{\mathcal{P}}_{p c h, 1}$ and using the similar arguments above, we can prove that $\overline{\mathcal{P}}_{p c h, 2}$ is a partial convex hull of $\overline{\mathcal{P}}$ where

$$
\begin{array}{rlrl}
\overline{\mathcal{P}}_{p c h, 2}:=\left\{T_{\omega} x+W_{\omega} y_{\omega} \geq r_{\omega},\right. & \omega \in \Omega, \\
& \left\|E_{\omega}^{j} y_{\omega}^{j}+F_{\omega}^{j} x-h_{\omega}^{j}\right\|_{p} \leq d_{\omega, 0}^{j}, & j \in J, \omega \in \Omega \\
& \left\|\bar{E}_{\omega_{1}, l}^{j} y_{\omega_{1}}^{j}+\bar{F}_{\omega_{1}, l}^{j} x-\bar{h}_{\omega_{1}, l}^{j}\right\|_{p} \leq d_{\omega_{1}, 0}^{j}, & l \in \mathcal{L}, j \in J, \\
& \left\|\bar{E}_{\omega_{2}, l}^{j} y_{\omega_{2}}^{j}+\bar{F}_{\omega_{2}, l}^{j} x-\bar{h}_{\omega_{2}, l}^{j}\right\|_{p} \leq d_{\omega_{2}, 0}^{j}, & l \in \mathcal{L}, j \in J, \\
& x \in X, y_{\omega_{1}}^{j} \in \mathbb{R}^{q}, y_{\omega_{2}}^{j} \in \mathbb{R}^{q}, y_{\omega}^{j} \in \mathbb{Z}^{q}, & \left.j \in J, \omega \in \Omega \backslash\left\{\omega_{1}, \omega_{2}\right\}\right\} .
\end{array}
$$

We repeat the foregoing steps by replacing $\overline{\mathcal{P}}$ by $\overline{\mathcal{P}}_{p c h, i}$ for $\omega_{i}, i=2, \ldots,|\Omega|-1$. Meanwhile, for each step, we assume that $\operatorname{conv}\left(\overline{\mathcal{K}}_{\omega_{i}}^{1}(x)\right)=\operatorname{Proj}_{y}\left(\overline{\mathcal{K}}_{t i g h t}^{\omega_{i}}\right)$ for all $x \in X$. Finally, we have $\operatorname{Proj}_{x, y, d}\left(\mathcal{P}_{p c h,|\Omega|}\right)=\operatorname{Proj}_{x, y, d}\left(\mathcal{P}_{p c h}\right)$ is a partial convex hull of $\mathcal{P}$. This completes the proof.

\section{Appendix B: Tight second stage formulations for structured TSS-CMIPs}

\section{Appendix B.1: Proof of Corollary 1}

For $x \in X$ and $\omega \in \Omega$, by substituting $\sigma=y_{\omega, 1}-f_{\omega} x \in \mathbb{Z}, \beta=h_{\omega}, v=y_{\omega, 2} \in \mathbb{R}_{+}$and $\rho_{0}=d_{\omega, 0} \in \mathbb{R}_{+}$in the set $\bar{Z}$, we get $\overline{\mathcal{K}}_{\omega}(x)$. Hence according to Proposition 2 , the convex hull of the feasible region of $\overline{\mathcal{Q}}_{\omega}(x)$, i.e., $\operatorname{conv}\left(\overline{\mathcal{K}}_{\omega}(x)\right)$, for all $x \in X$ can be obtained by adding inequality,

$$
\sqrt{\left(\left(1-2 \mu_{\omega}\right)\left(y_{\omega, 1}+f_{\omega} x-h_{\omega}\right)+\mu_{\omega}\right)^{2}+\left(y_{\omega, 2}\right)^{2}} \leq d_{\omega, 0},
$$

to the continuous relaxation of $\overline{\mathcal{K}}_{\omega}(x)$. Furthermore, we reformulate $\operatorname{con} v\left(\overline{\mathcal{K}}_{\omega}(x)\right)$ in higher dimensional space by adding variables $d_{\omega, 1}$ and $d_{\omega, 2}$, and obtain

$$
\begin{aligned}
& \left\{\left(y_{\omega, 1}, y_{\omega, 2}, d_{\omega, 1}, d_{\omega, 2}, d_{\omega, 0}\right) \in \mathbb{R} \times \mathbb{R}_{+}^{4}:\left|y_{\omega, 1}+f_{\omega} x-h_{\omega}\right| \leq d_{\omega, 1},\right. \\
& \left.\left|y_{\omega, 2}\right| \leq d_{\omega, 2}, \sqrt{d_{\omega, 1}^{2}+d_{\omega, 2}^{2}} \leq d_{\omega, 0}, \quad\left(1-2 \mu_{\omega}\right)\left(y_{\omega, 1}-\left\lfloor h_{\omega}\right\rfloor+f_{\omega} x\right)+\mu_{\omega} \leq d_{\omega, 1}\right\},
\end{aligned}
$$

which is the convex hull of the feasible region of $\mathcal{Q}_{\omega}(x)$, i.e., $\operatorname{conv}\left(\mathcal{K}_{\omega}(x)\right)$. 


\section{Appendix C: Partial convex hull for extensive formulation of structured} TSS-CMIPs

\section{Appendix C.1: Proof of Corollary 2}

For $x \in X$ and $\omega \in \Gamma$, let

$$
\begin{aligned}
\overline{\mathcal{K}}_{\text {tight }}^{\omega}(x)= & \left\{\left(y_{\omega, 1}, y_{\omega, 2}, d_{\omega, 0}\right) \in \mathbb{R} \times \mathbb{R}_{+}^{2}:(69)\right. \text { and } \\
& \left.\sqrt{\left(\left(1-2 \mu_{\omega}\right)\left(y_{\omega, 1}+f_{\omega} x-h_{\omega}\right)+\mu_{\omega}\right)^{2}+\left(y_{\omega, 2}\right)^{2}} \leq d_{\omega, 0}\right\} .
\end{aligned}
$$

From Corollary 1, we know that $\operatorname{conv}\left(\overline{\mathcal{K}}_{\omega}(x)\right)=\overline{\mathcal{K}}_{\text {tight }}^{\omega}(x)$ for all $x \in X$ and $\omega \in \Gamma$. Hence, by utilizing Theorem 5 , a partial convex hull of $\overline{\mathcal{P}}$ is given by $\overline{\mathcal{P}}_{p c h}$.

\section{Appendix C.2: Proof of Corollary 3}

For $x \in X$ and $\omega \in \Gamma$, let

$$
\begin{aligned}
\overline{\mathcal{K}}_{\text {tight }}^{\omega}(x)= & \left\{\left(y_{\omega}, d_{\omega, 0}\right) \in \mathbb{R} \times \mathbb{R}_{+}^{|J|}:\right. \text { (33), (34), and } \\
& \left.\left\|\bar{E}_{\omega, l}^{j} y_{\omega}^{j}+\bar{F}_{\omega, l}^{j} x-\bar{h}_{\omega, l}^{j}\right\|_{p} \leq d_{\omega, 0}^{j}, l \in \mathcal{L}, j \in J\right\},
\end{aligned}
$$

where the $i$ th row of $\bar{E}_{\omega, l}^{j} y_{\omega}^{j}+\bar{F}_{\omega, l}^{j} x-\bar{h}_{\omega, l}^{j}$ is either $y_{\omega}^{j}+f_{\omega, i}^{j} x-h_{\omega, i}^{j}$ or $\left(1-2 \mu_{\omega, i}^{j}\right)$ $\left(y_{\omega}^{j}-\left\lfloor h_{\omega, i}^{j}\right\rfloor+f_{\omega, i}^{j} x\right)+\mu_{\omega, i}^{j}$, and $\mu_{\omega, i}^{j}=h_{\omega, i}^{j}-\left\lfloor h_{\omega, i}^{j}\right\rfloor$. From Theorem 6, we know that $\operatorname{conv}\left(\overline{\mathcal{K}}_{\omega}(x)\right)=\overline{\mathcal{K}}_{\text {tight }}^{\omega}(x)$ for all $x \in X$ and $\omega \in \Gamma$ when $p=1$. Hence, by utilizing Theorem 5, it is clear that $\overline{\mathcal{P}}_{p c h}$ is a partial convex hull of $\overline{\mathcal{P}}$.

\section{Appendix C.3: Proof of Corollary 4}

For $x \in X$ and $\omega \in \Gamma$, let

$$
\begin{aligned}
\overline{\mathcal{K}}_{\text {tight }}^{\omega}(x) & =\left\{\left(y_{\omega}, d_{\omega, 0}\right) \in \mathbb{R}^{q} \times \mathbb{R}_{+}^{|J|}:\right. \text { (53) and (54) } \\
& \left.\left\|\bar{E}_{\omega, l}^{j} y_{\omega}^{j}+\bar{F}_{\omega, l}^{j} x-\bar{h}_{\omega, l}^{j}\right\|_{p} \leq d_{\omega, 0}^{j}, \quad l \in \mathcal{L}, j \in J\right\},
\end{aligned}
$$

where the $i$ th row of $\bar{E}_{\omega, l}^{j} y_{\omega}^{j}+\bar{F}_{\omega, l}^{j} x-\bar{h}_{\omega, l}^{j}$ is either $e_{\omega, i}^{j} y_{\omega}^{j}+f_{\omega, i}^{j} x-h_{\omega, i}^{j}$ or $\left(1-2 \mu_{\omega, i}^{j}\right)$ $\left(e_{\omega, i}^{j} y_{\omega}^{j}-\left\lfloor h_{\omega, i}^{j}\right\rfloor+f_{\omega, i}^{j} x\right)+\mu_{\omega, i}^{j}$, and $\mu_{\omega, i}^{j}=h_{\omega, i}^{j}-\left\lfloor h_{\omega, i}^{j}\right\rfloor$. From Theorem 7, we know $\operatorname{conv}\left(\overline{\mathcal{K}}_{\omega}(x)\right)=\overline{\mathcal{K}}_{\text {tight }}^{\omega}(x)$ for all $x \in X$ and $\omega \in \Gamma$ when $p=1$. Hence, by utilizing Theorem 5, we can claim that $\overline{\mathcal{P}}_{p c h}$ is a partial convex hull of $\overline{\mathcal{P}}$. 


\section{Appendix D: Deterministic polyhedral conic mixed integer sets}

Since this paper is focused on stochastic programming, we provide proofs of Theorems 6 and 7 in the manuscript, and utilize these results to provide the following proofs for Theorems 1-3 (which are important in their own right).

\section{Appendix D.1: Proof of Theorem 1}

In the proof of Theorem 6 , by substituting $W_{\omega}, y_{\omega}, r_{\omega}-T_{\omega} x, f_{\omega, i}^{j} x-h_{\omega, i}^{j}, d_{\omega, 1}^{j}, \ldots, d_{\omega, m_{2}}^{j}$, for $j=1, \ldots,|J|$, by $\mathcal{A}, \sigma, b, \beta_{i k}$, and $\rho_{1}^{k}, \ldots, \rho_{m}^{k}$, for $k=1, \ldots, K$, respectively, the set $\mathcal{K}_{\omega}^{1}(x)$ reduces to $R_{K}^{m}$. Additionally, $\operatorname{conv}\left(\mathcal{K}_{\omega}^{1}(x)\right)=\operatorname{conv}\left(R_{K}^{m}\right)$ reduces to the set defined as in Theorem 1.

\section{Appendix D.2: Proof of Theorem 2}

(a) In the proof of Theorem 7, we substitute $W_{\omega}, y_{\omega}, r_{\omega}-T_{\omega} x, E_{\omega}^{j}, f_{\omega, i}^{j} x-h_{\omega, i}^{j}$, and $d_{\omega, i}^{j}$, for $i=1, \ldots, m_{2}$ and $j=1, \ldots,|J|$, by $\mathcal{A}, \sigma, b, \mathcal{G}_{k}, \beta_{i k}$, and $\rho_{i}^{k}$, for $i=1, \ldots, m$ and $k=$ $1, \ldots, K$, respectively. This reduces the set $\mathcal{K}_{\omega}^{1}(x)$ to $S_{K}^{m, n}$ and $\operatorname{conv}\left(\mathcal{K}_{\omega}^{1}(x)\right)=\operatorname{conv}\left(S_{K}^{m, n}\right)$ reduces to the set obtained by adding

$$
\left(1-2 \beta_{i k}^{(1)}\right)\left(\sum_{t=1}^{n} g_{k t}^{i} \sigma_{k t}-\left\lfloor\beta_{i k}\right\rfloor\right)+\beta_{i k}^{(1)} \leq \rho_{i}^{k}, i=1, \ldots, m, k=1, \ldots, K,
$$

to the continuous relaxation of $S_{K}^{m, n}$.

(b) We prove this result using Corollary 4 in which we set $|\Omega|=|\Gamma|=1$ and $X=\mathbb{Z}^{u}$, and substitute variables $x, y_{\omega}^{j}$, and $d_{\omega, 0}^{j}$ for $j=1, \ldots,|J|$, by $\eta, \sigma^{k}$, and $\rho_{0}^{k}$ for $k=1, \ldots, K$, respectively, and parameters $T_{\omega}, W_{\omega}, r_{\omega}, E_{\omega}^{j}, F_{\omega}^{j}$, and $h_{\omega}^{j}$ by $\mathcal{A}_{1}, \mathcal{A}_{2}, b, \mathcal{G}^{k}, \mathcal{C}^{k}$, and $\beta_{k}$, respectively. Then similar to the previous proof, by reformulating $\overline{\mathcal{P}}$ and $\overline{\mathcal{P}}_{p c h}$ using additional continuous variables $\rho_{i}^{k}$ for $i=1, \ldots, m$, and $k=1, \ldots, K$ (as discussed in Sect. 4), we get $U_{K}^{m, n, u}$ and $U_{K, p c h}^{m, n, u}$, respectively. Since $\overline{\mathcal{P}}_{p c h}$ is a partial convex hull of $\overline{\mathcal{P}}$ according to Corollary 4, it is clear that $U_{K, p c h}^{m, n, u}$ is a partial convex hull of $U_{K}^{m, n, u}$.

\section{Appendix D.3: Proof of Theorem 3}

This result can be easily proved using Corollary 3 in which we set $|\Omega|=|\Gamma|=1$ and $X=\mathbb{Z}^{u}$, and substitute variables $x, y_{\omega}^{j}$, and $d_{\omega, 0}^{j}$ for $j=1, \ldots,|J|$, by $\eta, \sigma^{k}$, and $\rho_{0}^{k}$ for $k=1, \ldots, K$, respectively, and parameters $T_{\omega}, W_{\omega}, r_{\omega}, F_{\omega}^{j}$, and $h_{\omega}^{j}$ by $\mathcal{A}_{1}, \mathcal{A}_{2}, b, \mathcal{C}^{k}$, and $\beta_{k}$, respectively. Then, by reformulating $\overline{\mathcal{P}}$ and $\overline{\mathcal{P}}_{p c h}$ using additional continuous variables $\rho_{i}^{k}$ for $i=1, \ldots, m$, and $k=1, \ldots, K$ (as discussed in Sect. 4), we get $T_{K}^{m, u}$ and $T_{K, p c h}^{m, u}$, respectively. Since $\overline{\mathcal{P}}_{p c h}$ is a partial convex hull of $\overline{\mathcal{P}}$ according to Corollary 3 , it is clear that $T_{K, p c h}^{m, u}$ is a partial convex hull of $T_{K}^{m, u}$. 


\section{References}

1. Ahmed, S., Tawarmalani, M., Sahinidis, N.V.: A finite branch-and-bound algorithm for two-stage stochastic integer programs. Math. Program. 100(2), 355-377 (2004)

2. Ahmed, S., Garcia, R., Kong, N., Ntaimo, L., Parija, G., Qiu, F., Sen, S.: Siplib: A stochastic integer programming test problem library. https://www2.isye.gatech.edu/ sahmed/siplib/ (2015)

3. Atamtürk, A., Narayanan, V.: Conic mixed-integer rounding cuts. Math. Program. 122(1), 1-20 (2010)

4. Atamtürk, A., Narayanan, V.: Lifting for conic mixed-integer programming. Math. Program. 126(2), 351-363 (2011)

5. Bansal, M.: Facets for single module and multi-module capacitated lot-sizing problems without backlogging. Discrete Appl. Math. 255, 117-141 (2019)

6. Bansal, M., Kianfar, K.: $n$-step cycle inequalities: facets for continuous $n$-mixing set and strong cuts for multi-module capacitated lot-sizing problem. Math. Program. 154, 113-144 (2015)

7. Bansal, M., Kianfar, K.: Facets for continuous multi-mixing set with general coefficients and bounded integer variables. Discrete Optim. 26, 1-25 (2017)

8. Bansal, M., Huang, K.L., Mehrotra, S.: Decomposition algorithms for two-stage distributionally robust mixed binary programs. SIAM J. Optim. 28(3), 2360-2383 (2018)

9. Bansal, M., Huang, K.L., Mehrotra, S.: Tight second stage formulations in two-stage stochastic mixed integer programs. SIAM J. Optim. 28(1), 788-819 (2018)

10. Beasley, J.E.: Or-library: capacitated warehouse location. http://people.brunel.ac.uk/ mastjjb/jeb/orlib/ capinfo.html (1988)

11. Ben-Tal, A., den Hertog, D., De Waegenaere, A., Melenberg, B., Rennen, G.: Robust solutions of optimization problems affected by uncertain probabilities. Manag. Sci. 59(2), 341-357 (2012)

12. Benders, J.F.: Partitioning procedures for solving mixed-variables programming problems. Numer. Math. 4(1), 238-252 (1962)

13. Bertsimas, D., Popescu, I.: Optimal inequalities in probability theory: a convex optimization approach. SIAM J. Optim. 15(3), 780-804 (2005)

14. Bertsimas, D., Doan, X.V., Natarajan, K., Teo, C.P.: Models for minimax stochastic linear optimization problems with risk aversion. Math. Oper. Res. 35(3), 580-602 (2010)

15. Bodur, M., Dash, S., Günlük, O., Luedtke, J.: Strengthened benders cuts for stochastic integer programs with continuous recourse. INFORMS J. Comput. 29(1), 77-91 (2016)

16. Breton, M., El Hachem, S.: Algorithms for the solution of stochastic dynamic minimax problems. Comput. Optim. Appl. 4(4), 317-345 (1995)

17. Breton, M., Hachem, S.E.: A scenario aggregation algorithm for the solution of stochastic dynamic minimax problems. Stoch. Stoch. Rep. 53(3-4), 305-322 (1995)

18. Calafiore, G.: Ambiguous risk measures and optimal robust portfolios. SIAM J. Optim. 18(3), 853-877 (2007)

19. Carøe, C.C., Tind, J.: A cutting-plane approach to mixed 0-1 stochastic integer programs. Eur. J. Oper. Res. 101(2), 306-316 (1997)

20. Cezik, M., Iyengar, G.: Cuts for mixed 0-1 conic programming. Math. Program. 104(1), 179-202 (2005)

21. Cook, W., Kannan, R., Schrijver, A.: Chvátal closures for mixed integer programming problems. Math. Program. 47(1-3), 155-174 (1990)

22. Delage, E., Ye, Y.: Distributionally robust optimization under moment uncertainty with application to data-driven problems. Oper. Res. 58, 595-612 (2010)

23. Dupacová, J.: The minimax approach to stochastic programming and an illustrative application. Stochastics 20, 73-88 (1987)

24. Gade, D., Kücükyavuz, S., Sen, S.: Decomposition algorithms with parametric gomory cuts for two-stage stochastic integer programs. Math. Program. 1-26 (2012)

25. Günlük, O., Linderoth, J.: Perspective reformulations of mixed integer nonlinear programs with indicator variables. Math. Program. 124(1), 183-205 (2010)

26. Jiang, R., Guan, Y.: Risk-averse two-stage stochastic program with distributional ambiguity. Oper. Res. 66(5), 1390-1405 (2018)

27. Khan, M.R., Lewis, D.A.: A network model for nursing staff scheduling. Zeitschrift für Oper. Res. 31(6), B161-B171 (1987)

28. Kianfar, K., Fathi, Y.: Generalized mixed integer rounding inequalities: facets for infinite group polyhedra. Math. Program. 120(2), 313-346 (2009)

29. Kilınc-Karzan, F.: On minimal valid inequalities for mixed integer conic programs. Math. Oper. Res. 41(2), 477-510 (2016)

30. Kim, K., Mehrotra, S.: A two-stage stochastic integer programming approach to integerated staffing and scheduling with application to nurse management. Oper. Res. 63(6), 1431-1451 (2015) 
31. Kong, N., Schaefer, A.J., Hunsaker, B.: Two-stage integer programs with stochastic right-hand sides: a superadditive dual approach. Math. Program. 108(2-3), 275-296 (2006)

32. Kong, N., Schaefer, A.J., Ahmed, S.: Totally unimodular stochastic programs. Math. Program. 138(1-2), 1-13 (2013)

33. Kücükyavuz, S., Sen, S.: An introduction to two-stage stochastic mixed-integer programming. Lead. Dev. INFORMS Commun. INFORMS TutOR. Oper. Res. 1, 1-27 (2017)

34. Laporte, G., Louveaux, F.V.: The integer L-shaped method for stochastic integer programs with complete recourse. Oper. Res. Lett. 13(3), 133-142 (1993)

35. Love, D.K., Bayraksan, G.: Phi-divergence constrained ambiguous stochastic programs for data-driven optimization. Tech. rep., Department of Integrated Systems Engineering, The Ohio State University, Columbus, Ohio. http://www.optimization-online.org/DB_HTML/2016/03/5350.html (2015)

36. Luo, F., Mehrotra, S.: A decomposition method for distributionally-robust two-stage stochastic mixedinteger cone programs. Available at arXiv:1911.08713

37. Mehrotra, S., Zhang, H.: Models and algorithms for distributionally robust least squares problems. Math. Program. 148(1-2), 123-141 (2014)

38. Mijangos, E.: An algorithm for two-stage stochastic mixed-integer nonlinear convex problems. Ann. Oper. Res. 235(1), 581-598 (2015)

39. Miller, A.J., Wolsey, L.A.: Tight formulations for some simple mixed integer programs and convex objective integer programs. Math. Program. 98(1-3), 73-88 (2003)

40. Modaresi, S., Vielma, J.P.: Convex hull of two quadratic or a conic quadratic and a quadratic inequality. Math. Program. 164(1-2), 383-409 (2017)

41. Modaresi, S., Kilinc, M.R., Vielma, J.P.: Split cuts and extended formulations for mixed integer conic quadratic programming. Oper. Res. Lett. 43(1), 10-15 (2015)

42. Nemhauser, G.L., Wolsey, L.A.: Integer and Combinatorial Optimization. Wiley, New York, USA (1988)

43. Nemhauser, G.L., Wolsey, L.A.: A recursive procedure to generate all cuts for $0-1$ mixed integer programs. Math. Program. 46(1-3), 379-390 (1990)

44. Ntaimo, L.: Disjunctive decomposition for two-stage stochastic mixed-binary programs with random recourse. Oper. Res. 58(1), 229-243 (2009)

45. Ozaltin, O.Y., Prokopyev, O.A., Schaefer, A.J.: Two-stage quadratic integer programs with stochastic right-hand sides. Math. Program. 133(1), 121-158 (2012)

46. Pflug, G., Wozabal, D.: Ambiguity in portfolio selection. Quant. Finance 7(4), 435-442 (2007)

47. Pflug, G., Pichlera, A., Wozabalb, D.: The $1 / n$ investment strategy is optimal under high model ambiguity. J. Bank. Finance 36(2), 410-417 (2012)

48. Prékopa, A.: Stochastic Programming. Kluwer Academic, Boston (1995)

49. Rebman, K.R.: Total unimodularity and the transportation problem: a generalization. Linear Algebra Appl 8(1), 11-24 (1974)

50. Riis, M., Andersen, K.A.: Applying the minimax criterion in stochastic recourse programs. Eur. J. Oper. Res. 165(3), 569-584 (2005)

51. Romeijnders, W., Morton, D.P., Van Der Vlerk, M.H.: Assessing the quality of convex approximations for two-stage totally unimodular integer recourse models. INFORMS J. Comput. 29(2), 211-231 (2017)

52. Sanjeevi, S., Masihabadi, S., Kianfar, K.: Using cuts for mixed integer knapsack sets to generate cuts for mixed integer polyhedral conic sets. Math. Program. 159(1), 571-583 (2016)

53. Scarf, H.: A min-max solution of an inventory problem. Stud. Math. Theory Invent. Prod. 12, 201-209 (1958)

54. Schultz, R., Stougie, L., Van Der Vlerk, M.H.: Solving stochastic programs with integer recourse by enumeration: a framework using gröbner basis. Math. Program. 83(1-3), 229-252 (1998)

55. Sen, S., Higle, J.L.: The $C^{3}$ theorem and a $D^{2}$ algorithm for large scale stochastic mixed-integer programming: set convexification. Math. Program. 104(1), 1-20 (2005)

56. Sen, S., Sherali, H.D.: Decomposition with branch-and-cut approaches for two-stage stochastic mixedinteger programming. Math. Program. 106(2), 203-223 (2006)

57. Sherali, H.D., Fraticelli, B.M.: A modification of benders' decomposition algorithm for discrete subproblems: an approach for stochastic programs with integer recourse. J. Glob. Optim. 22(1-4), 319-342 (2002)

58. Sherali, H.D., Zhu, X.: On solving discrete two-stage stochastic programs having mixed-integer first-and second-stage variables. Math. Program. 108(2-3), 597-616 (2006)

59. Sun, R.R., Shylo, O.V., Schaefer, A.J.: Totally unimodular multistage stochastic programs. Oper. Res. Lett. 43(1), 29-33 (2015)

60. Vinel, A., Krokhmal, P.: On valid inequalities for mixed integer p-order cone programming. J. Optim. Theory Appl. 160(2), 439-456 (2014) 
61. Wang, Z., Glynn, P.W., Ye, Y.: Likelihood robust optimization for data-driven problems. Comput. Manag. Sci. 13(2), 241-261 (2016)

62. Wozabal, D.: A framework for optimization under ambiguity. Ann. Oper. Res. 193(1), 21-47 (2012)

63. Yanıkoğlu, I., den Hertog, D.: Safe approximations of ambiguous chance constraints using historical data. INFORMS J. Comput. 25(4), 666-681 (2012)

64. Zhao, C., Guan, Y.: Data-driven risk-averse two-stage stochastic program with $\zeta$-structure probability metrics. http://www.optimization-online.org/DB_HTML/2015/07/5014.html (2015)

Publisher's Note Springer Nature remains neutral with regard to jurisdictional claims in published maps and institutional affiliations. 